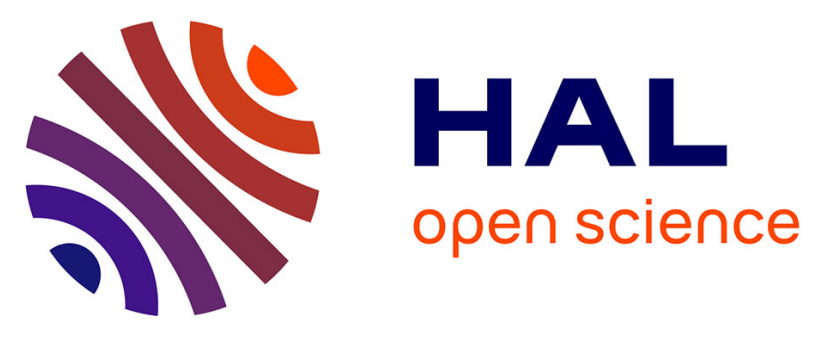

\title{
Optimized Design of Multi-MHz Frequency Isolated Auxiliary Power Supply for Gate Drivers in Medium-Voltage Converters
}

Ole Christian Spro, Pierre Lefranc, Sanghyeon Park, Juan M. Rivas-Davila, Dimosthenis Peftitsis, Ole-Morten Midtgard, Tore Undeland

\section{To cite this version:}

Ole Christian Spro, Pierre Lefranc, Sanghyeon Park, Juan M. Rivas-Davila, Dimosthenis Peftitsis, et al.. Optimized Design of Multi-MHz Frequency Isolated Auxiliary Power Supply for Gate Drivers in Medium-Voltage Converters. IEEE Transactions on Power Electronics, 2020, 35 (9), pp.9494-9509. 10.1109/TPEL.2020.2972977 . hal-03260242

\section{HAL Id: hal-03260242 \\ https://hal.science/hal-03260242}

Submitted on 29 Sep 2021

HAL is a multi-disciplinary open access archive for the deposit and dissemination of scientific research documents, whether they are published or not. The documents may come from teaching and research institutions in France or abroad, or from public or private research centers.
L'archive ouverte pluridisciplinaire HAL, est destinée au dépôt et à la diffusion de documents scientifiques de niveau recherche, publiés ou non, émanant des établissements d'enseignement et de recherche français ou étrangers, des laboratoires publics ou privés. 


\title{
Optimized Design of Multi-MHz Frequency Isolated Auxiliary Power Supply for Gate Drivers in Medium Voltage Converters
}

\author{
Ole Christian Spro, Student Member, IEEE, Pierre Lefranc, Sanghyeon Park, Student Member, IEEE, \\ Juan M. Rivas-Davila, Senior Member, IEEE, Dimosthenis Peftitsis, Senior Member, IEEE, \\ Ole-Morten Midtgård, Member, IEEE, and Tore Undeland, Fellow, IEEE
}

\begin{abstract}
This paper presents the design and optimisation of a suitable topology for an isolated DC-DC auxiliary power supply with high isolation voltage and low coupling capacitance. The converter consists of a GaN HEMT inverter operating at $6.78 \mathrm{MHz}$, an LCC resonance tank and a class $\mathrm{E}$ low dv/dt rectifier. Furthermore, the galvanic isolation is implemented using a coreless planar transformer that enables higher insulation voltage with similar or better converter efficiency compared to designs using magnetic material. An analytical design methodology is developed, however, SPICE investigations show that optimal designs might lie outside the validity of the design equations. Consequently, a virtual prototyping tool is developed based on a genetic algorithm with numerical simulations and, in turn, is used to optimise the converter. The optimisation algorithm maximises the converter efficiency while minimising the transformer size. Prototypes are constructed based on the resulting Pareto front. Experimental results show the validity of the simulated results. Prototypes transferring power up to $15 \mathrm{~W}$ with a peak efficiency of $81 \%$ are shown. The selected topology enables insulating voltages exceeding $40 \mathrm{kV}$ and coupling capacitances below $10 \mathrm{pF}$.
\end{abstract}

Index Terms-Resonant power conversion, Inductive power transmission, Virtual prototyping, Medium voltage, Gate drivers

\section{INTRODUCTION}

With the continuous development of high voltage power semiconductors, medium voltage converters can obtain lower power losses and increased efficiency. Main applications for medium voltage (up to $36 \mathrm{kV}_{\mathrm{RMS}}$ ) converters include industrial drives (e.g. fans, mills, pumps), wind turbines with fullyrated converters, medium voltage dc distribution grids for marine vessels, urban transportation or other applications, and flexible ac transmission systems (FACTS) used in medium voltage grids [1]-[7]. Such converters operate with dc-links of up to a few tens of kilovolts, which necessitates the use of either modular multilevel converter (MMC) topologies, or 2-level or 3-level voltage source converters (VSC) using

O. C. Spro, D. Peftitsis, O.-M. Midtgård and T. Undeland are with the Norwegian University of Science and Technology. (email: olechristian.spro@ntnu.no).

P. Lefranc is with Univ. Grenoble Alpes, CNRS, Grenoble INP*, G2Elab, F-38000 Grenoble, France, *Institute of Engineering Univ. Grenoble Alpes

S. Park and J. Rivas-Davila are with Stanford University at 420 Via Palou Mall, Stanford, CA, 94305.

The authors acknowledges the funds received through the Norwegian Research Council grant 243711 and the travel scholarship from The Fund of the Norwegian Institute of Technology. valves with series-connected power semiconductor switches (Figs. 1a and 1b). The characteristics and current development of high voltage $\mathrm{SiC}$ devices make them especially attractive for medium voltage applications.

Regardless of the specific medium voltage converter topology, an auxiliary power supply is always required for energising control and gate circuitry. In particular, the auxiliary power supply provides power to a switching unit that consists either of a submodule in a MMC converter or several seriesconnected switches in 2-level VSCs (see Fig. 1). The typical output power requirement of the auxiliary power supply is approximately $10 \mathrm{~W}$ [8, pp. 121-132].

One way to supply this power is through the local medium voltage dc-link by using a DC-DC step-down converter, such as the tapped inductor Buck converter [8], [9]. However, to improve system safety and provide better diagnostics, it is more beneficial to supply auxiliary circuits via an external power source [10]. The latter requires the design of sophisticated isolated power supplies with high-voltage insulation and low coupling capacitance. The insulation requirement is related to the operating voltage of the medium voltage converter, requiring an isolation voltage of at least the full dc-link voltage. A crucial challenge is to simultaneously minimise the coupling capacitance of the auxiliary power supply, as this capacitor constitutes a path for the noise current generated during $\mathrm{dv} / \mathrm{dt}$ events and possibly affecting control circuitry [11]. Moreover, the fast switching capabilities of SiC MOSFETs increase the importance of this requirement.

\section{A. Isolated power supplies}

Isolated power supplies feeding gate drivers in medium voltage converters is targeted in several publications. In general, these works can be categorised along two dimensions; a single converter feeding a single load or multiple loads, and transformer design with or without magnetic material. For most of the concepts, a resonant or quasi-resonant converter structure is used. Within resonant converter topologies, the seriesseries compensated (SS) resonant converter structure is usually preferred due to its simple design. An established architecture consists of a single converter feeding multiple loads using a single primary winding that goes through several toroidal magnetic cores on which the secondary turns are winded and supplies the loads separately [12]-[14]. The reported coupling 


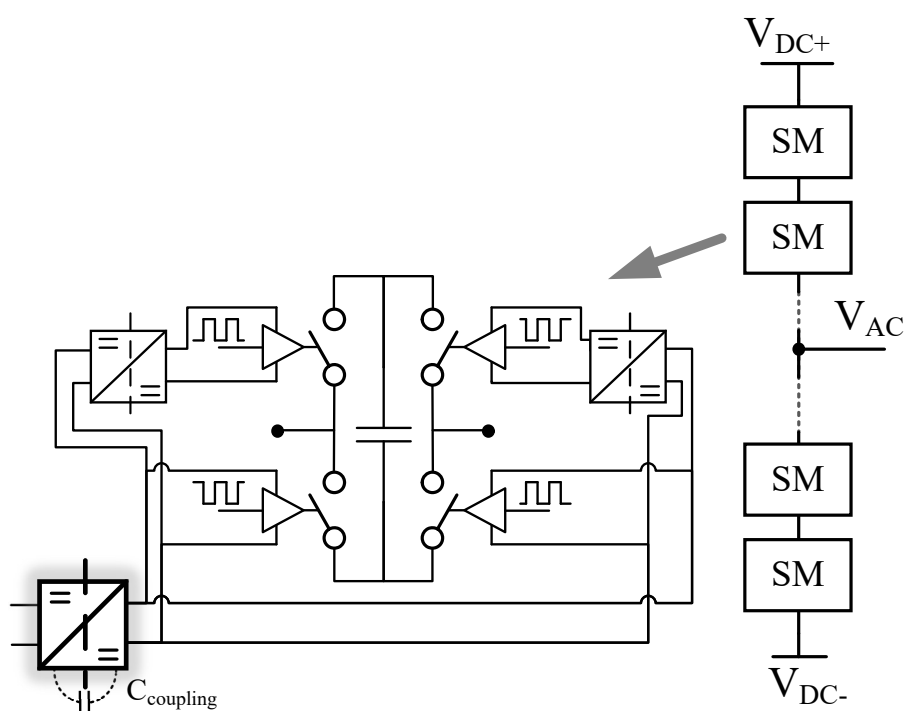

(a)

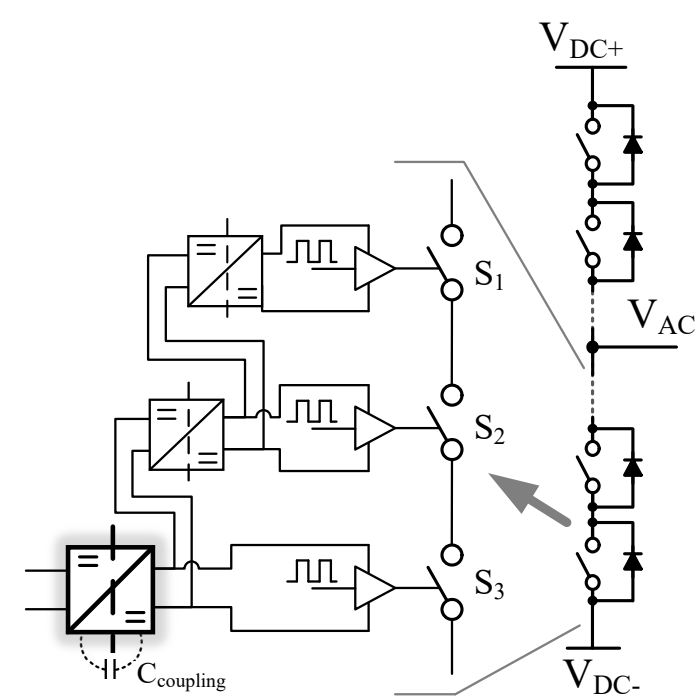

(b)

Fig. 1. Conceptual diagrams of the application - supplying power to the gate drivers in a medium voltage converter arm. The arms of the medium voltage converter consist of either a) submodules (SM) in a multilevel converter or b) series-connected switches. The auxiliary power supply targeted in the paper is shown in bold.

capacitance is in the range of $1-2 \mathrm{pF}$ [13], which is very low. The disadvantage with a single to multiple load system is that the medium voltage converter system is dependent on the reliability of the single feeding converter. Furthermore, the previously mentioned feature of better diagnostics is lost.

An alternative architecture consists of separate feeding converters, each with its own transformer and supplying each load separately [10], [15]-[17]. A compact transformer concept designed for up to $36 \mathrm{kV}_{\mathrm{RMS}}$ application have been shown using magnetic material and a dielectric design using potting gel and conductive paint [10]. Although the withstand voltage and small system size are impressive, the transformer concept is complex to manufacture. In comparison, a transformer concept that is easier to fabricate with a breakdown voltage higher than $40 \mathrm{kV}$ is demonstrated in [15], [16]. Here, the transformer with a magnetic core has an airgap of $1 \mathrm{~mm}$ in which a nonmagnetic insulating material is placed. However, it is reported that any increase in the gap distance, and hence the insulation voltage, drastically decreases the converter efficiency [15].

A more traditional transformer design consisting of insulated wires with different magnetic core materials and shapes has been proposed in [17], [18]. Additionally, an insulating material is wrapped around the core to increase the insulation voltage. Such schemes have been shown to provide an insulation voltage above $20 \mathrm{kV}$ with low coupling capacitances of less than $5 \mathrm{pF}$. Yet, such layering of dielectric material must be designed carefully to avoid partial discharges in air pockets between layers. Furthermore, such systems have to comply with several criteria in the standards of the International Electrotechnical Commission (IEC) governing insulation schemes which may result in de-rating of the insulation voltage [18].

The auxiliary power supply with galvanic isolation can also be established by using power-over-fibre technology [19]-[21]. Although a very low coupling capacitance can be achieved, this technology faces power transmission limitations and low efficiency.

Several publications have also investigated the use of inductive power transfer (IPT) systems as a method of fulfilling the insulation requirements [22]-[25]. Both single converter to single load [22], [25] and single converter to multiple loads [23], [24] have been demonstrated. IPT systems uses resonant converters to transfer power by coreless transformers that have poor coupling coefficients. The gap between the primary and secondary sides of the transformer is used to establish an insulation barrier by using either air or another non-magnetic material as the insulation material. Intrinsically, large gap distances result in low coupling capacitance. IPT systems should operate at high frequency to limit the physical size of the transformer and passive components.

\section{B. Contribution of this paper}

This paper presents an isolated auxiliary power supply which is based on the insulation scheme reported by $\mathrm{Am}$ et al. [15]. By using GaN devices and together with a coreless planar transformer, the presented converter can improve the efficiency while simultaneously increasing the achievable insulation voltage compared to the published work. The proposed topology in this paper is shown in Fig. 2. It consists of a half bridge inverter, a LCC resonant tank, a coreless transformer and a class $\mathrm{E}$ rectifier designed for an operating frequency of $6.78 \mathrm{MHz}$. The proposed topology results in a simple system that can operate in open loop with large variations in load.

Furthermore, an analytical design method is proposed for the topology. In addition, further investigations reveal that the rectifier performance improves when designed for frequencies beyond that of the resonance converter using SPICE simulations. Above-resonance operation is found to be beneficial for the efficiency, however, this necessitates simulation-based design. Thus, the paper demonstrates the feasibility of using a virtual prototyping tool for the converter design which is 
used to optimise the converter efficiency while minimising the transformer coupling capacitance. The optimisation results are coherent with the presented design theory and investigations. Three potential candidates for the non-magnetic insulation material are explored in the paper and the insulation performance is evaluated in terms of insulation voltage and coupling capacitance.

The paper is organised as follows. Section II describes the converter topology and implementation choices. In addition, arguments for the use of a computer assisted design method are given. In Section III, the converter modelling is analysed and then verified experimentally. The optimisation tool and results are presented in Section IV. Section V presents experimental results of four prototypes taken from the optimised designs obtained in Section IV. Finally, the conclusions are drawn in Section VI.

\section{Converter Structure for the Auxiliary Power SUPPLY}

\section{A. Proposed Converter Structure}

In the literature, a SS resonant converter structure is usually preferred due to the simple design procedure and that the phase angle for the converter does not change with load resistance [26]. The output voltage of the resonant system is rectified using a full bridge diode rectifier consisting of four diodes. In comparison, the class $\mathrm{E}$ low $\mathrm{dv} / \mathrm{dt}$ rectifier consists of a single diode. While the rectifier topology is known in the literature for several decades [27, Chapter 4] [28], it has gained interest in recent years for use in IPT systems [29], [30]. For this topology, the transformer secondary side coil, $L_{s e c}$, and the parasitic capacitance of the diode are used as part of the passive elements needed to form a resonant soft switching circuit. This results in a very compact and efficient rectifier for multi-MHz systems. The diode losses are lower than for full bridge diode rectifier solutions that are reported as a significant contribution to total losses [25], especially for low output-power designs.

There are several inverter topologies available for multi$\mathrm{MHz}$ (i.e. $6.78 \mathrm{MHz}$ and beyond) frequency applications. Due to the high switching frequency, the inverter must operate with soft switching in order to eliminate the excessive switching losses. The class E inverter [27, Chapter 13] [31], [32] and the class $\Phi_{2}$ [33] are often used, both consisting of a single switch. Main drawbacks of these converters are low switch utilisation and sensitivity to load variation and tuning issues. In this work, a half-bridge structure is used in combination with a LCC resonant tank [34], which results in a total component count that is similar to the SS topology (summarised in Table I). The main difference is the need for a discrete inductor, while only a single diode is needed. In a half bridge, the switch voltage is equal to the dc-link voltage, leading to high switch utilisation. This allows for using an integrated half bridge module with GaN high electron mobility transistors (HEMT) that is available for low power applications [35]. Using GaN HEMT devices operating at high frequency keeps the converter size small while ensuring low driving losses. The characteristic capacitances of GaN HEMTs are lower than for $\mathrm{Si}$ and $\mathrm{SiC}$
TABLE I

COMPONENT COMPARISON BETWEEN THE SS TOPOLOGY AND THE PROPOSED TOPOLOGY

\begin{tabular}{ccccc}
\hline & Capacitors & Discrete inductors & Diodes & Switches \\
\hline Series-series & 2 & 0 & 4 & 2 \\
Proposed & 3 & 1 & 1 & 2 \\
\hline
\end{tabular}

counterparts with similar rating [36]. In this regard, GaN HEMT is an enabling technology as using $\mathrm{Si}$ or $\mathrm{SiC}$ devices at $\mathrm{MHz}$ frequency would result in driver losses comparable to the output power of the auxiliary power supply. This is critical for low power applications. However, a concern regarding GaN devices is the effect of dynamic on-resistance [37], [38] and losses related to the charging and discharging of the output capacitance [39].

\section{B. Analytical Design Method}

The design method for the converter is developed based on the fundamental harmonic approximation [27]. The rectifier is modelled as shown in Fig. 3a. The load resistance is set as

$$
R_{\text {load,min }}=\frac{V_{\text {out }}^{2}}{P_{\text {nom }}}
$$

where $V_{\text {out }}$ is the output voltage at the nominal power, $P_{\text {nom }}$. Next, the required inductance and capacitance of the rectifier, $L_{\text {sec }}$ and $C_{\text {rect }}$ are found by

$$
\begin{aligned}
& L_{\text {sec }}=\frac{R_{\text {load } \min }}{\omega_{\text {sec }} Q_{r}} \\
& C_{\text {rect }}=\frac{1}{\omega_{\text {sec }}^{2} L_{\text {sec }}}
\end{aligned}
$$

where $Q_{r}$ is the loaded quality factor [27]. $\omega_{s e c}$ is often set equal to the operating frequency, $\omega_{0}$. Furthermore, the voltage transfer function between the rectifier input voltage and the $\mathrm{dc}$ output voltage of the rectifier is defined as

$$
M_{V}=\frac{V_{\text {out }}}{V_{m}}
$$

where $V_{m}$ is the amplitude of the voltage source at the rectifier input [27]. Furthermore, the coupled inductors of the primary and secondary side are modelled as (Fig. 3c).

$$
\begin{aligned}
& V_{\text {prim }}=-j \omega_{0} M I_{\text {sec }} \\
& V_{\text {sec }}=-j \omega_{0} M I_{\text {prim }}
\end{aligned}
$$

where the mutual inductance, $\mathrm{M}$, is defined as

$$
M=k \sqrt{L_{\text {prim }} L_{\text {sec }}}
$$

where $k$ is the transformer coupling factor. The transformer efficiency depends on the coupling coefficient and the quality factor of the coils. For two spiral coils, the coupling coefficient is at its highest when the coils are of the same size with identical winding number [40]. Consequentially, the coil inductance is chosen as $L_{\text {prim }}=L_{\text {sec }}=L$. 


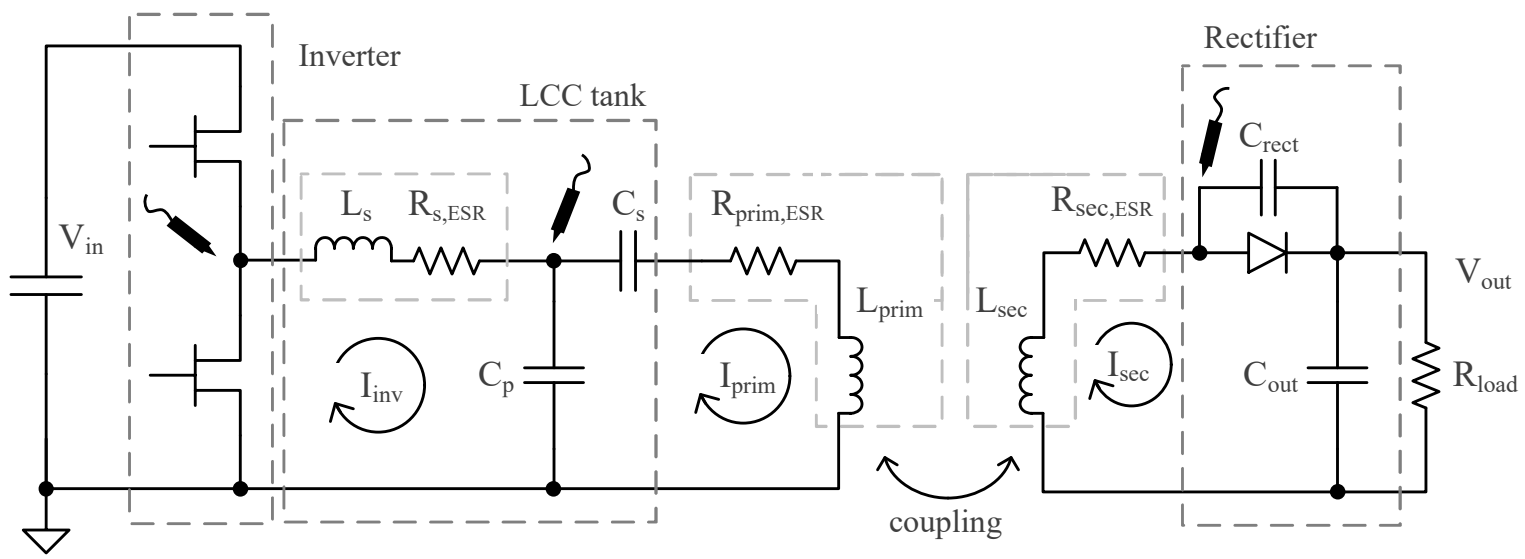

Fig. 2. Schematic diagram of the converter topology consisting of a half bridge inverter, an LCC resonance tank, coreless transformer and a class E rectifier. The probe signals indicate the voltage measurement points in the experimental setup for time domain comparison.

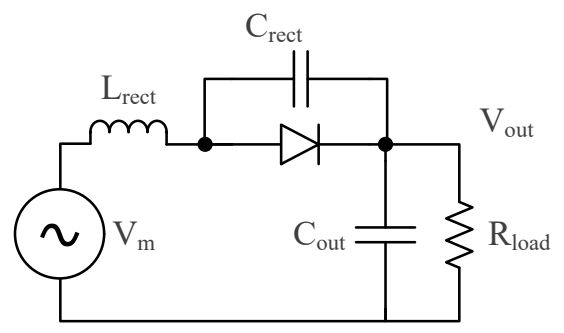

(a)

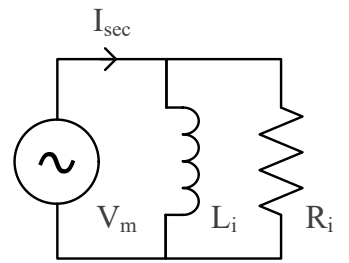

(b)

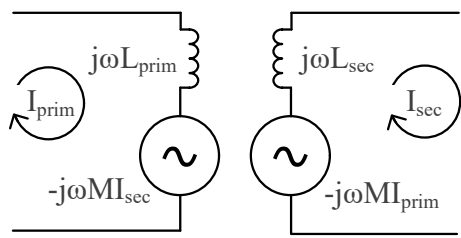

(c)

Fig. 3. Schematic diagrams of a) the rectifier model, b) the equivalent input impedance of the rectifier and c) the coupled inductor model.

Starting from Eq. (6), the current in the primary side of the transformer is dimensioned to satisfy the required input voltage of the rectifier, $V_{m}$, as defined in Eq. (4).

$$
I_{\text {prim }}=\frac{V_{m}}{\omega_{0} M}=\frac{V_{m}}{\omega_{0} k L}
$$

Next, the equations for the primary side circuit are developed by using Kirchhoff's voltage law for the fundamental frequency.

$$
\begin{array}{r}
\frac{\sqrt{2}}{\pi} V_{i n}=\left(j \omega L_{s}-j \frac{1}{\omega C_{p}}\right) I_{i n v}+j \frac{1}{\omega C_{p}} I_{\text {prim }} \\
0=j \frac{1}{\omega C_{p}} I_{i n v}+I_{\text {prim }}\left(j \omega L_{\text {prim }}-j \frac{1}{\omega C_{p}}+Z_{\text {load }}\right)
\end{array}
$$

where $Z_{\text {load }}=R_{\text {load }}+j X_{\text {load }}$ is the rectifier impedance reflected to the primary side, $V_{i n}$ is the dc input voltage, $C_{p}$ is the parallel capacitor, $L_{s}$ is the series inductance, $L_{\text {prim }}$ is the transformer primary side inductance, and $I_{i n v}$ and $I_{\text {prim }}$ are the currents as indicated in Fig. 2. Next, we set the resonance of $L_{s}$ and $C_{p}$ according to the following criteria:

$$
\omega_{0}^{2}=\frac{1}{L_{s} C_{p}}=\left(2 \pi f_{s w}\right)^{2}
$$

where $\omega_{0}$ is the series resonance of $L_{s}$ and $C_{p}$, and $f_{s w}$ is the switching frequency of the inverter. With Eq. (11) fulfilled, the part containing $I_{i n v}$ in Eq. (9) equals zero and the expression for current $I_{\text {prim }}$ becomes

$$
I_{\text {prim }}=-j \omega_{0} C_{p} \frac{\sqrt{2}}{\pi} V_{\text {in }}
$$

Hence, the current in the primary side of the transformer can be dimensioned by $C_{p}$ for a given dc-link voltage and is approximately constant over the converter operating range. Combining Eqs. (8) and (12), the value of $C_{p}$ is found

$$
C_{p}=\frac{\pi V_{m}}{2 \omega_{0}^{2} k L V_{i n}}
$$

In the next step, the series capacitor, $C_{s}$, is used to tune the switching current. The switching current, $I_{i n v, s w}$, is defined as the current amplitude of $I_{i n v}$ at the moment of switching in the half bridge. This current is the sum of the fundamental and higher order harmonics of current resulting from the square wave voltage output of the inverter [34]. At the moment of switching, the switching current equals

$$
I_{\text {inv }, s w}=\frac{\sqrt{2} V_{\text {in }}}{\pi Z_{c}}\left(\frac{L_{\text {prim }}}{L_{s}}-\frac{C_{p}}{C_{s}}+\frac{X_{\text {load }}}{Z_{c}}-\frac{\pi^{2}}{8}\right)
$$

where $I_{i n v, s w}$ is the output current of the inverter at the moment of switching, $Z_{c}$ is the characteristic impedance of the primary resonance circuit $\left(Z_{c}=\sqrt{L_{s} / C_{p}}\right)$ and $X_{\text {load }}$ is the reactance of the rectifier reflected to the primary side. As described in [34], ZCS can be achieved by setting this expression equal to zero. However, ZCS entails loss of the $C_{O S S}$ energy for every switching transition, which is substantial at multi-MHz frequencies. If $I_{i n v, s w}$ is set to a negative value, then ZVS can be obtained. Thus, for a given transformer design, $C_{s}$ governs the size of current at the switching instant. $C_{s}$ is chosen so that the impedance seen from the inverter stays inductive, and with a small switching 
current that allows for soft switching within the set dead time of the half bridge. Since $R_{\text {load }}$ does not appear in Eq. (14), this switching current is not dependent on the active power transfer to the secondary side. However, Eq. (14) contains the term $X_{\text {load }}$. The equivalent impedance of the class E rectifier used in this paper has shown to change with duty cycle, and hence with the operating point [28]. This is countered by setting a margin for the switching current.

From Eq. (5) and using Fig. 3b, the rectifier impedance reflected to the primary side is found as

$$
Z_{\text {load }}=-\frac{w_{0}^{2} M^{2}}{R_{i}}-\frac{w_{0} M^{2}}{L_{i}}
$$

where $R_{i}$ and $L_{i}$ are the equivalent input resistance and inductance as defined in [27]. The switching current is set according to the output capacitance of the inverter and the target dead time.

$$
I_{\text {inv }, s w}=-\frac{C_{O S S} V_{i n}}{t_{\text {commutation }}}
$$

where $C_{O S S}$ is the output capacitance of the inverter switches and $t_{\text {commutation }}$ is the time required to complete a switching transient which is equal or smaller than the deadtime. Finally, the value of $C_{s}$ is found by combining Eq. (14), Eq. (15) and Eq. (16)

$$
C_{s}=\frac{C_{p}}{\frac{\pi Z_{c} I_{\text {inv }, s w}}{\sqrt{2} V_{i n}}+\frac{L_{\text {prim }}}{L_{s}}+\frac{X_{\text {load }}}{Z_{c}}-\frac{\pi^{2}}{8}}
$$

An example of dimensioning the converter is given in the Appendix. The SPICE simulation of the converter shows that the output voltage is within $2.6 \%$ of the ideal case.

\section{Design of an above-resonance rectifier}

The converter is further investigated by changing the resonance frequency of the rectifier, $\omega_{s e c}$. In [29], many class $\mathrm{E}$ rectifiers are compared according to their design parameters. It is shown that designing the rectifier off-resonance might give advantageous characteristics for the system. An example of a benefit is a smaller output voltage variation with varying load current if the rectifier resonance frequency is higher than operating frequency, $\omega_{0}$ [29]. The rectifier circuit shown in Fig. $3 \mathrm{a}$ is evaluated through SPICE simulations using ideal components. The relation between the resonance frequency of the rectifier and the voltage source frequency is defined as

$$
A_{r}=\frac{\omega_{s e c}}{\omega_{0}}
$$

The rectifier is dimensioned using Eq. (1), Eq. (2) and Eq. (3), for which $Q_{r}$ is set so that the diode duty cycle is 50\% [27], [29]. $A_{r}$ is varied in this work from 1.5 to 2.0 since this has been found to be the most promising design space in terms of high efficiency and lowest output voltage variation [29]. With the component values set, the load resistor is varied from $R_{L, \text { min }}$ and towards open circuit (a decreasing load). $M_{V}$ is shown in Fig. 4a. The trend in $M_{V}$ for nominal output power is consistent with the findings in [29]. For $A_{r}$ equal to 1.5 (and below), the rectifier output is closer to a current source behaviour. For higher $A_{r}$, the output behaves like a voltage source until approximately $10 \%$ load. In Fig. $4 \mathrm{~b}$ the $M_{V}$ is used to investigate the expected variation in output voltage with changing load. Low voltage variation is observed for higher $A_{r}$. On the other hand, this lower variation comes at a price of lower efficiency since $M_{V}$ decreases. Additionally, the second harmonic of the rectifier circuit increases drastically as shown in Fig. 4c. Nevertheless, an added benefit of increasing $A_{r}$ is that the required inductor value decreases. Fig. 4 d shows the passive component values for varying $A_{r}$. The inductance value decreases from 2.41 to $0.164 \mu \mathrm{H}$, which makes the converter size smaller. The increase in capacitance value has a softer impact on the practical implementation.

However, for the optimisation problem, it is paramount that the model used in the optimisation method is coherent with the final experimental results. Fig. 4c shows that there is substantial content of harmonics and, in consequence, the fundamental harmonic approximation can no longer be assumed accurate. In this paper, it is rather argued for choosing a computer assisted design process due to the complexity and interactions between design parameters. A high fidelity simulation model serves as the basis for the optimisation algorithm. Although this approach requires more time due to the development time of exact models and long simulation times, the benefit is that the inverter, transformer and rectifier are optimised holistically. The converter topology contains many components resulting in many degrees of freedom. Moreover, even the resonance frequency could be chosen different from the switching frequency, adding to the design problem complexity. To globally answer the design problem, a virtual prototyping tool is used that is based on a genetic algorithm that is suitable for a mixed integer programming problem. However, since the output of the optimisation process is dependent on the fidelity of the simulation results, accurate simulation models must be first established.

\section{Modelling, Simulation PROCEDURE AND VERIFICATION OF CONVERTER OPERATION}

A simulation model of the converter structure was established in a SPICE simulation software $\left(\right.$ LTSpice ${ }^{\mathrm{TM}}$ ), as such simulators are suitable for detailed component behaviour of the semiconductor devices. The electrical simulation model is used to investigate the converter behaviour in time domain when steady state operation has been established. Appropriate semiconductor components for the application are selected among commercially available components, and their SPICE models are included in the simulation. Furthermore, all passive components are added as shown in Fig. 2. For all capacitors, the equivalent series resistance is set according to the manufacturer data for the chosen operating frequency. For the inductances, the calculated or measured value for the series resistance was used.

The coreless transformer was modelled using finite element software as a magneto-static problem. From this model, lumped parameter model of the transformer can be extracted to be used in the electrical simulation in time domain. A free software for solving electromagnetic problems using finite 


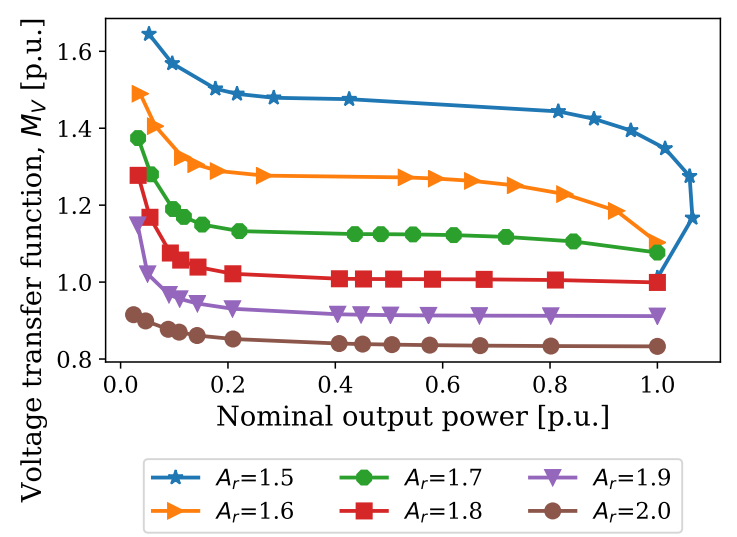

(a)

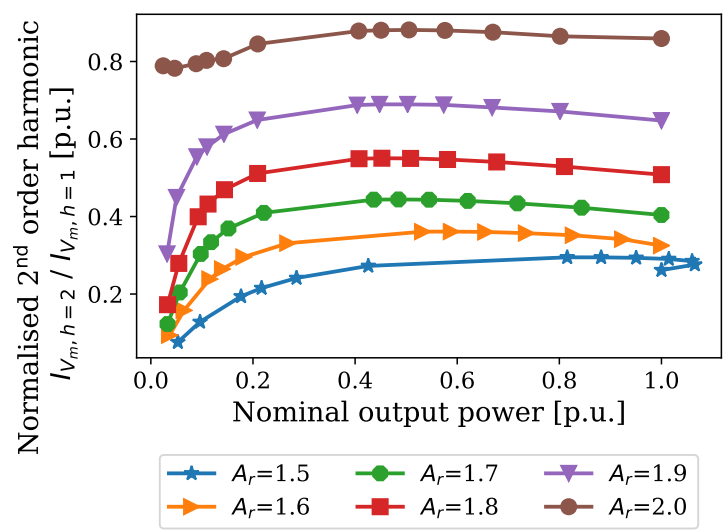

(c)

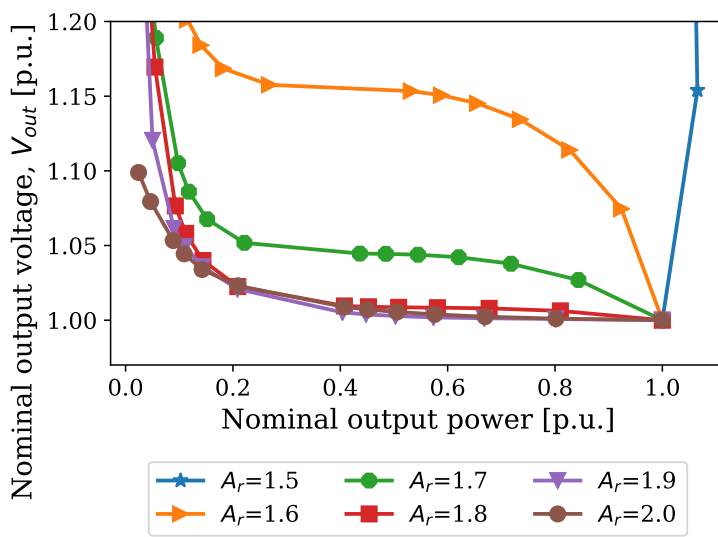

(b)

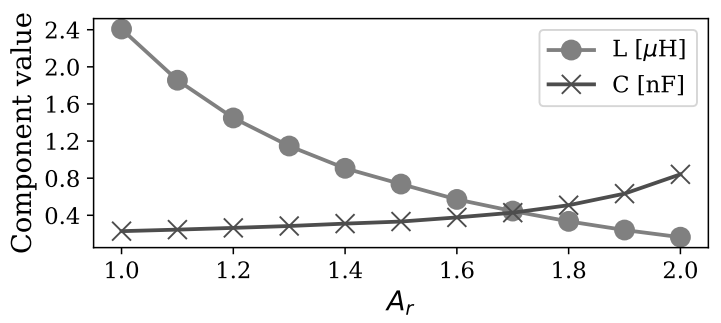

(d)

Fig. 4. Results from SPICE simulations of the rectifier circuit assuming ideal components. The a) voltage transfer function, b) output voltage variation and c) relative 2 nd harmonic in the current are plotted as a function of output power. d) shows the values for the resonance components.

element method, FEMM, was used [41]. The transformer is designed as spiral inductors, thus simplifying the electromagnetic problem to a $2 \mathrm{D}$ geometry with axisymmetrical properties. Additionally, the transformer can be accurately reproduced by printing windings on a PCB. Figure 5a shows the geometry of the transformer windings and the magneto-static problem. An example simulation result showing the lines of the magnetic field distribution using FEMM is shown in Fig. 5b.

An initial prototype is constructed to verify the results obtained in simulation. Both the converter prototype and the measurement setup are seen in Fig. 6. The prototype is designed to be able to handle the resulting currents when operating with an input voltage of $48 \mathrm{~V}$, resulting in the prototype component values that are given in Table II. The inverter is realised using a GaN HEMT half bridge module (TI LMG5200). As source for the logic input signals for the lower and upper switch, a signal generator is used (Tektronix AFG3052C). $C_{p}$ is chosen to obtain a circulating current of approximately $2 \mathrm{~A}$ at $48 \mathrm{~V}$ input voltage. The transformer was designed with a 5:2 turn ratio, which results in an output voltage of approximately $20 \mathrm{~V}$. On the rectifier side, a $\mathrm{SiC}$ Schottky diode is used in the rectifier (STPSC406B, $600 \mathrm{~V} / 4 \mathrm{~A}$ ).

To be able to evaluate the accuracy of the simulation model of the inverter, a loss estimation for the inverter is obtained thermally using a thermal camera (Fluke Ti25). The loss estimate takes into account the ambient temperature and is compensated for the driving losses. All prototype component values are measured with an impedance analyser (Agilent E4990). The estimated lumped parameters of the prototype PCB transformer are extracted and presented in Table III where they are compared to the transformer parameters from the FEMM simulation. As can be seen, the transformer inductance values are close to the values obtained from the simulation study. The additional inductance in the developed transformer design is hypothesised to originate from the connecting wires that are not accounted for in FEMM (see Fig. 5a). Hence, the measurements verify that simulated inductance values in FEMM can be realised in an experimental setup with high accuracy.

The converter is simulated and tested in the lab for several input voltages and load resistances. Fig. 7a shows the total system efficiency, the output power and inverter losses for an input voltage of $48 \mathrm{~V}$ while the load resistance varies from 15 to $1000 \Omega$. The peak measured efficiency is $67 \%$ and the converter obtains a peak output power of approximately $8 \mathrm{~W}$. The difference in the maximum efficiency between simulation and experimental values is approximately 5\%. Part of this error is due to the thermal and electrical model of the inverter used in the simulation. The comparison of the simulated and measured inverter losses in Fig. 7a shows that the inverter 


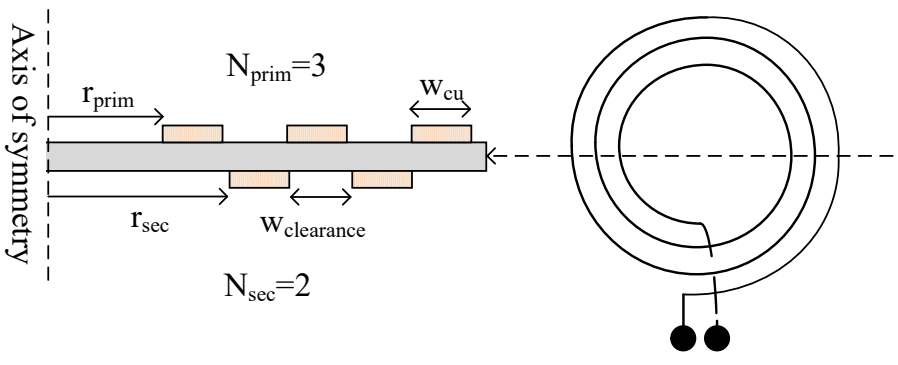

(a)

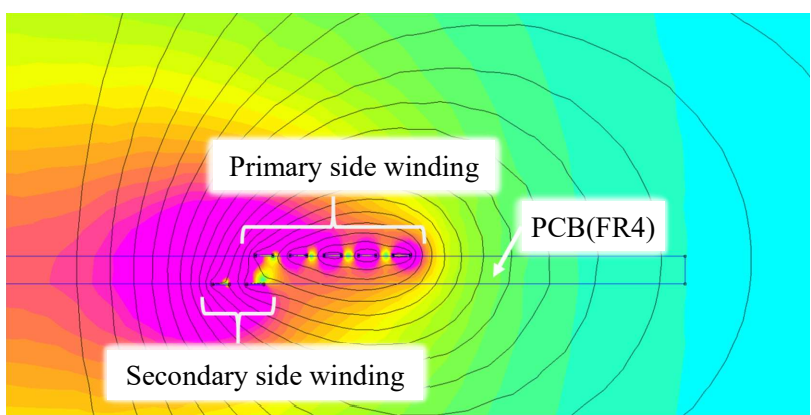

(b)

Fig. 5. Modelling of a coreless transformer printed on PCB. a) Axisymmetric geometry and FEM modelling of the PCB transformer cross section. b) Example view of magnetic density calculation for the transformer in FEMM.

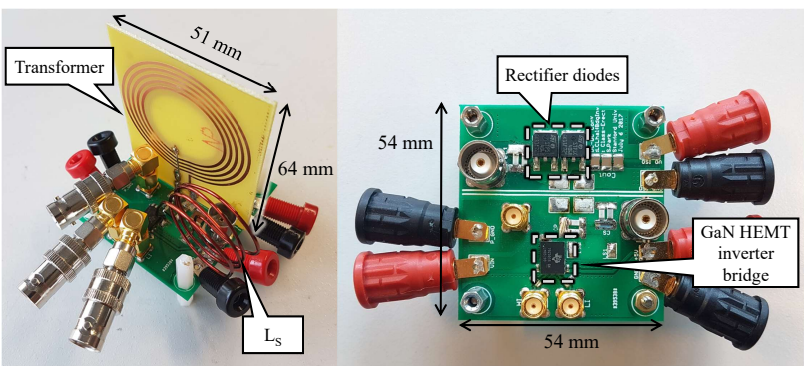

(a)

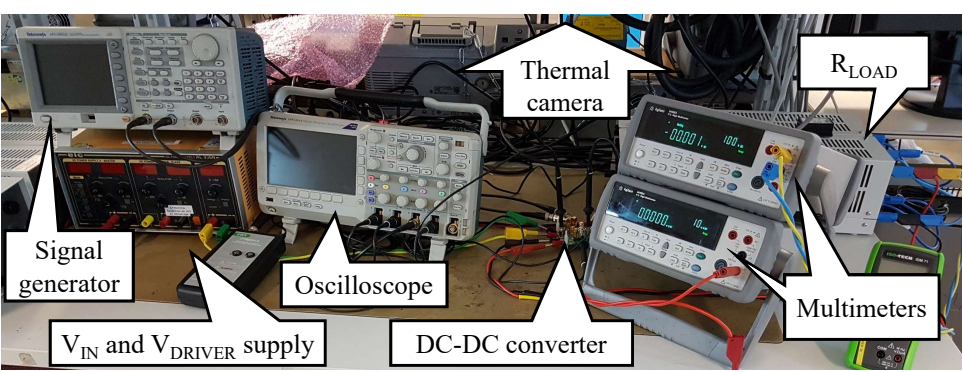

(b)

Fig. 6. Photo of a) the initial prototype featuring a coreless transformer made on a PCB and b) the test setup.

TABLE II

PARAMETERS OF THE PROTOTYPE CONVERTER USED FOR VALIDATION OF THE SIMULATION MODEL.

\begin{tabular}{lcccccccccc} 
Component & $\begin{array}{c}\text { Inverter } \\
-\end{array}$ & $L_{s}$ & $C_{p}$ & $C_{s}$ & $L_{\text {prim }}$ & $L_{\text {sec }}$ & coupling & $C_{\text {rect }}$ & $C_{\text {out }}$ & Diode \\
& - & $\mathrm{nH}$ & $\mathrm{pF}$ & $\mathrm{pF}$ & $\mathrm{nH}$ & $\mathrm{nH}$ & - & $\mathrm{pF}$ & $\mathrm{nF}$ & - \\
\hline Value & LMG5200 & 473 & 981 & 330 & 1379 & 240 & 0.48 & 330 & 100 & STPSC406B
\end{tabular}

TABLE III

COMPARISON OF TRANSFORMER CHARACTERISTICS BETWEEN FEMM SIMULATION AND THE ESTIMATED VALUES FOUND THROUGH IMPEDANCE MEASUREMENT.

\begin{tabular}{lccc}
\hline Parameter & $\begin{array}{c}\text { Value } \\
\text { FEM design }\end{array}$ & $\begin{array}{c}\text { Value } \\
\text { experimental }\end{array}$ & Error \\
\hline$L_{\text {prim }}$ & $1369 \mathrm{nH}$ & $1379 \mathrm{nH}$ & $1 \%$ \\
$L_{\text {sec }}$ & $210 \mathrm{nH}$ & $240 \mathrm{nH}$ & $15 \%$ \\
coupling & 0.48 & 0.48 & - \\
\hline
\end{tabular}

losses are underestimated in simulation. It is the hypothesis of the authors that these losses are related to two phenomena in the GaN HEMTs. One is the losses related to charging and discharging of the output capacitance, $C_{O S S}$. The second loss factor is dynamic on-state resistance, resulting in a higher effective on-resistance than stated in the device datasheet. Experimental data on hysteresis losses has been published recently [39], [42]. Depending on which manufacturer data from [39] is used for estimating the losses, a range in losses of 0.4 to $1 \mathrm{~W}$ is obtained per device. However, this is for $650 \mathrm{~V}$ devices. In [42], measurements of the output capacitance losses are performed for a $100 \mathrm{~V}$ device, indicating a loss factor of approximately $5-10 \%$ of the $C_{O S S}$ energy per cycle [42]. Using this relationship, the output capacitor loss for the two devices in the half bridge then evaluates to

$$
\begin{aligned}
P_{\text {CSS }} & =2 \cdot \%_{\text {percycle }} \cdot E_{\text {oss }} \cdot f \\
& =\%_{\text {percycle }} \cdot C_{O S S} \cdot V_{D S}^{2} \cdot f
\end{aligned}
$$

Using a conservative $\%_{\text {percycle }}=10 \%, C_{O S S}=266 \mathrm{pF}$ from the datasheet and $V_{d c}=48 \mathrm{~V}, P_{C_{O S S}}$ evaluates to approximately $0.42 \mathrm{~W}$. This power loss is added to the simulated inverter loss as a shaded area in Fig. 7a. Consequently, the gap between simulated and experimentally measured losses in the inverter is partly explained by the $C_{O S S}$ loss mechanism. Moreover, part of the additional losses are hypothesised to be due to dynamic on-resistance. This effect has been shown to affect GaN HEMTs. The magnitude of the increased effective resistance depends on the operating parameters. At $6.78 \mathrm{MHz}$ and $50 \%$ duty cycle, the on-period for each switch in the inverter is approximately $70 \mathrm{~ns}$, which is below the time frame of published work investigating the transient dynamic on-state resistance of commercially available devices. Yet, two recent 

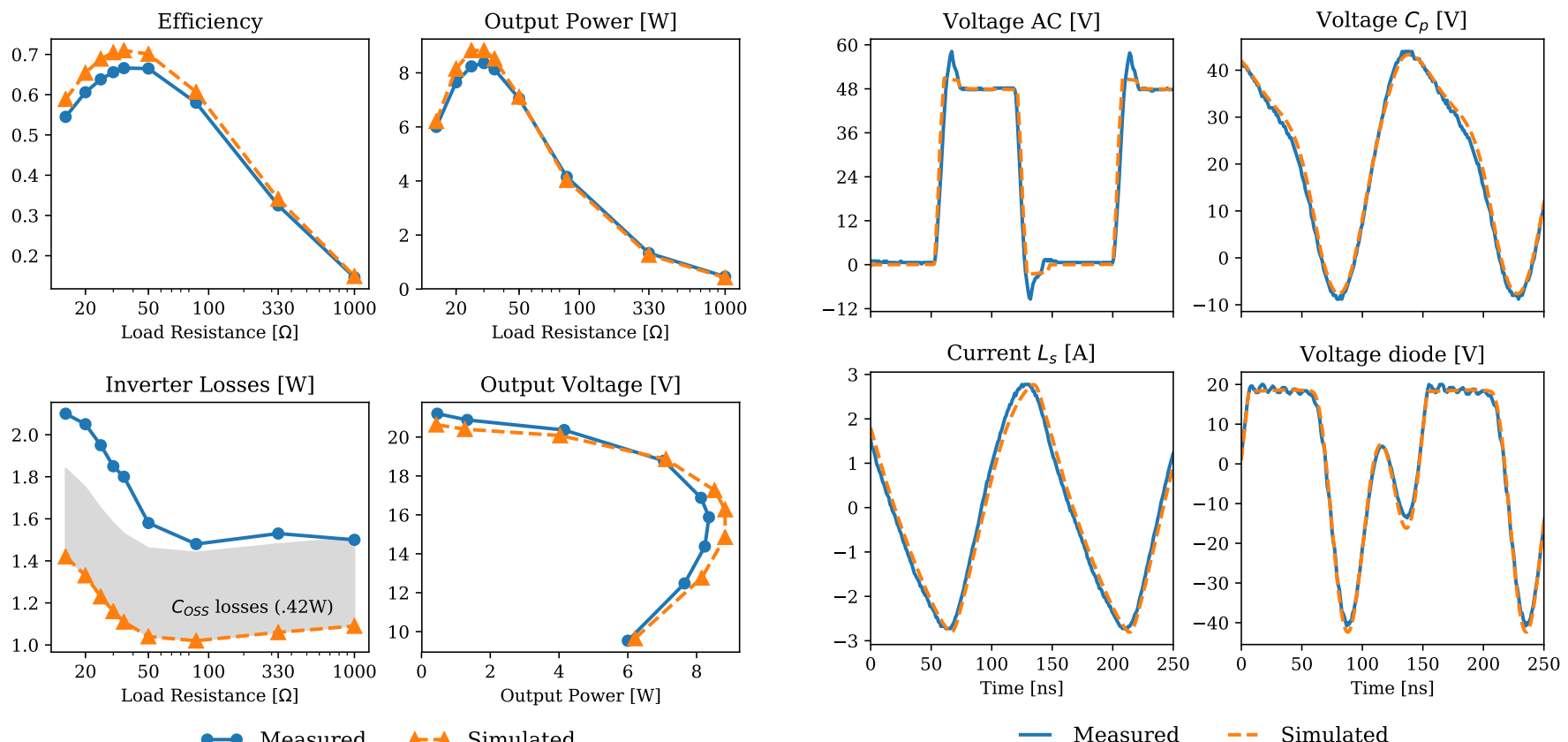

(a)

(b)

Fig. 7. Comparison of simulated and experimental results of the initial prototype operating with $48 \mathrm{~V}$ input voltage. The solid lines are the measured signals and the dashed lines are the simulated signals. a) DC-DC efficiency, output power and inverter losses with varying load resistance. Additionally, variation in output voltage with output power is also shown. b) Waveforms in time domain from the prototype operating with a $35 \Omega$ load.

works have investigated the effect on the average on-resistance in the megahertz range and with soft switching condition [43], [44]. The results from these works indicate that the effective resistance can be increased up to a factor of 7. In conclusion, the experimental results are expected to show higher losses, and hence, lower efficiency than the simulated results.

The simulated versus experimental performance of the converter is also investigated in time domain by four measurements. Three voltages are measured (shown in Fig. 2 by probe symbols) using passive probes (TPP0200) - the output voltage of the half bridge, the voltage over $C_{p}$ and the voltage at the anode of the diode. The probe tip capacitance is added in the simulation model to account for probe loading. In addition, the current in $L_{s}$ is measured with a Rogowski coil (PEM CWT015). An example of the waveform comparison is shown in Fig. 7b. A good accordance is observed between the simulation and the measured signals. A difference is seen at the edges related to the reverse conduction of the half bridge. In addition to the measured overshoot, a slight difference in the reverse conduction voltage drop of the simulation model and the GaN chip can be observed. For the three other waveforms, the voltages and the current deviate slightly during transients containing harmonics. Yet, the simulation is deemed well within the needed accuracy, knowing that some increased losses should be expected. The goal is to identify the potential of the selected topology. Using the simulation model as input, an optimisation method can be utilised to obtain designs with maximised efficiency. The resulting converter design could then be tested experimentally to establish an expectation of the final converter performance.

\section{CONVERTER OPTIMISATION}

This section describes the optimisation procedure and the experimental results. The goal of the optimisation algorithm is defined through the objective functions. For the present paper, two such functions are defined. The first function maximises the converter efficiency, while the second one minimises the size of the transformer. The transformer size is defined as the radius of the outer circumference of the transformer windings. The largest value of either the primary or secondary side is selected. Minimising the transformer size is an indirect method of minimising the transformer coupling capacitance without adding additional simulation steps to quantify the capacitance value. Furthermore, a constraint for the output voltage is added. The optimisation problem is thus formulated as follows:

$$
\begin{array}{ll}
\underset{\mathbf{x}}{\operatorname{maximize}} & O b j_{1}(\mathbf{x})=\eta_{d c-d c} \\
\underset{\mathbf{x}}{\operatorname{minimize}} & O b j_{2}(\mathbf{x})=\max \left(r_{\text {prim }}, r_{\text {sec }}\right) \\
\text { subject to } & \mathbf{x} \geq \mathbf{x}_{\text {lowerbound }} \\
& \mathbf{x} \leq \mathbf{x}_{\text {upperbound }} \\
& V_{\text {out }, \text { min }} \leq V_{\text {out }}(\mathbf{x}) \leq V_{\text {out }, \text { max }}
\end{array}
$$

where $\mathbf{x}$ is a vector of variables of the optimisation with minimum and maximum values as defined by $\mathbf{x}_{\text {lowerbound }}$ and $\mathbf{x}_{\text {upperbound }}, \eta_{d c-d c}$ is the converter efficiency, $r_{p r i m}$ and $r_{s e c}$ are the outer radius of the transformer primary and secondary sides respectively, and $V_{\text {out }}$ is the converter output voltage.

The optimisation algorithm can vary the geometry of the transformer and the remaining passive elements of the con- 


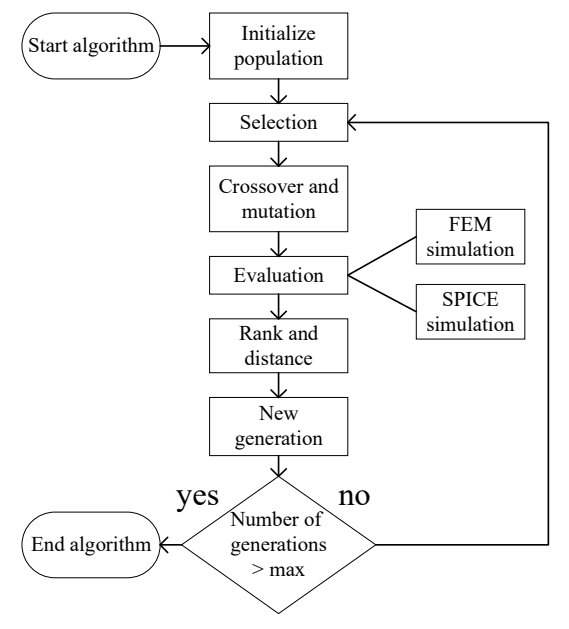

Fig. 8. Flow chart for the genetic algorithm used for the design optimisation.

verter. Changes to the transformer geometry results in variation in the lumped parameters, which in turn are used in the circuit simulation. In total, the multi-objective optimisation has 10 variables. The variable vector, $\mathbf{x}$, is hence defined as

$\mathbf{x}=\left(N_{\text {prim }}, N_{\text {sec }}, w_{\text {cu }}, r_{\text {prim }}, r_{\text {sec }}, L_{s}, C_{p}, C_{s}, C_{\text {rect }}, R_{\text {load }}\right)$

where $N_{\text {prim }}$ and $N_{\text {sec }}$ are the number of turns on the primary and secondary side respectively, $w_{c u}$ is the width of the copper track, $r_{\text {prim }}$ and $r_{\text {sec }}$ is the inner diameter of the primary and secondary respectively, and $L_{s}, C_{p}, C_{s}, C_{\text {rect }}$ and $R_{\text {load }}$ are the component values of the circuit as indicated in Fig. 2. The load resistance is defined as an optimisation variable to ensure that an optimal efficiency point is found for any given combination of values for the other passive elements.

A genetic algorithm is chosen to identify the Pareto front of the converter. The algorithm, NSGA-II, is suitable for the multi-objective, mixed integer programming problem [45]. Figure 8 shows the algorithm flow chart. Initialisation of the population is done using random sampling of all variables. During the evaluation step, each individual is evaluated in terms of efficiency and transformer size. First, the lumped parameters of the transformer are found by FEM simulation as described in the previous section. Following the FEM simulation, an LTSpice simulation is run with the circuit parameters of the individual to find the converter efficiency. The timedomain simulation runs for a sufficiently long time period so that steady-state conditions are reached. Since convergence cannot be guaranteed for all SPICE simulations, a time-out is set for each simulation. If the simulation does not finish in time, the individual is rejected by the algorithm. When both of the simulations are finished, the individuals are evaluated based on the objective functions. In addition, violation of the given constraints influences the evaluation of the individuals. The best individuals are chosen among the parents and the children to form the new generation. The algorithm continues to run until the generation number exceeds the maximum number set by the user.

For the optimisation procedure, a suitable use case is constructed that is based on the schematic diagram shown in
Fig. 1 and which is summarised in Table IV. An auxiliary power supply provides power to a switching unit that consists either of a module in a MMC converter or several seriesconnected switches. Regardless of the switching unit, the power required would be similar at approximately $10 \mathrm{~W}[8$, pp. 121-132]. The input voltage is set to a common dc bus voltage of $48 \mathrm{~V}$ while the output voltage constraint is set according to the driving voltage needed for the switches. For $\mathrm{SiC}$ MOSFETs, typical driving voltages are in the range of $-5-0 \mathrm{~V}$ negative driving voltage to $+15-20 \mathrm{~V}$ positive driving voltage. Hence, the output voltage constraint is set between $20-25 \mathrm{~V}$. As insulation material, FR4 material is used with a thickness of $1.5 \mathrm{~mm}$. This criterion is set to facilitate the production of the simulated transformers for prototyping. Future optimisations could include further details regarding the insulation scheme. The algorithm is launched with 100 individuals that are developed over 125 generations. Using a single processor, the simulation is carried out and the simulation time is approximately 3.5 days. The development of the optimisation with increasing generation number and the final generation are shown in Figs 9a and 9b.

The 100 individuals forming the Pareto front achieves a peak system efficiency ranging from $78 \%$ to above $87 \%$ with a transformer radius of approximately 10 to $17 \mathrm{~mm}$. It is observed that the efficiency obtained by the optimisation algorithm give substantially higher values than that for the initial prototype reported in Section III. The individuals are investigated in terms of average values of key parameters to identify the design trends that result in high efficiency. These values are presented in Table V. Common for all individuals is a transformer turns-ratio of $4: 4$ which maximises transformer coupling as discussed in Section II. Hence, the voltage conversion from $48 \mathrm{~V}$ input to $20-25 \mathrm{~V}$ output is achieved by different characteristics. It can be seen that the average resonance frequency of the rectifier, $\omega_{s e c}$, is approximately 1.5 times higher than the operating frequency, thus increasing the voltage transfer function towards the peak value. Again, this is in line with the investigations in Section II. Since the turns ratio is the same for all individuals, the increased transformer size is mainly due to wider windings that result in lowered track resistance. In turn, the transformer and system efficiency increases. Moreover, the average resonance frequency of the primary side series resonance, $\omega_{0}$, is slightly higher than operating frequency. This is contrary to the circuit design derived from the analytic approach, where the resonance frequency is set to the operating frequency. An important finding that effects the converter implementation is that the inductance of the transformer can be decreased compared to the existing design method in literature [27].

TABLE IV

USE CASE FOR THE OPTIMISATION ALGORITHM.

\begin{tabular}{ccccc}
\hline$V_{I N}$ & $V_{\text {out }, \text { min }}$ & $V_{\text {out }, \max }$ & $P_{\text {out }, \max }$ & Insulation \\
\hline $48 \mathrm{~V}$ & $20 \mathrm{~V}$ & $25 \mathrm{~V}$ & $\geq 10 \mathrm{~W}$ & $1.5 \mathrm{~mm}$ FR4 \\
\hline
\end{tabular}




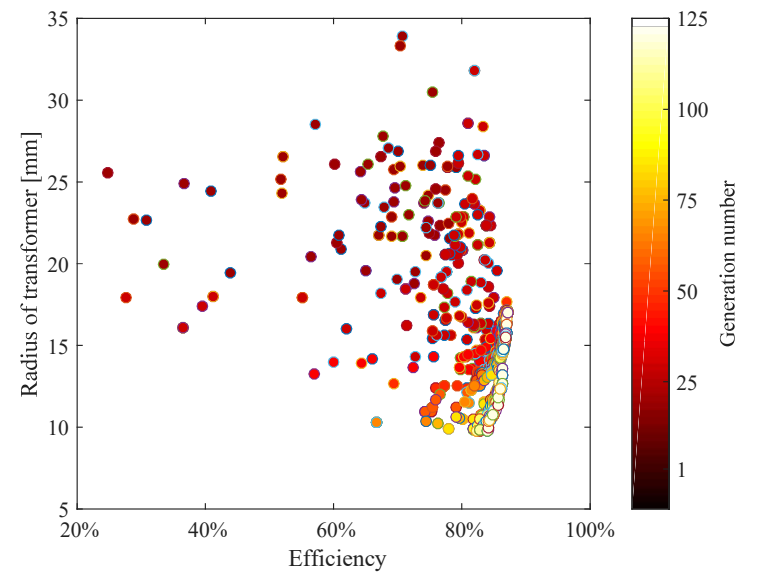

(a)

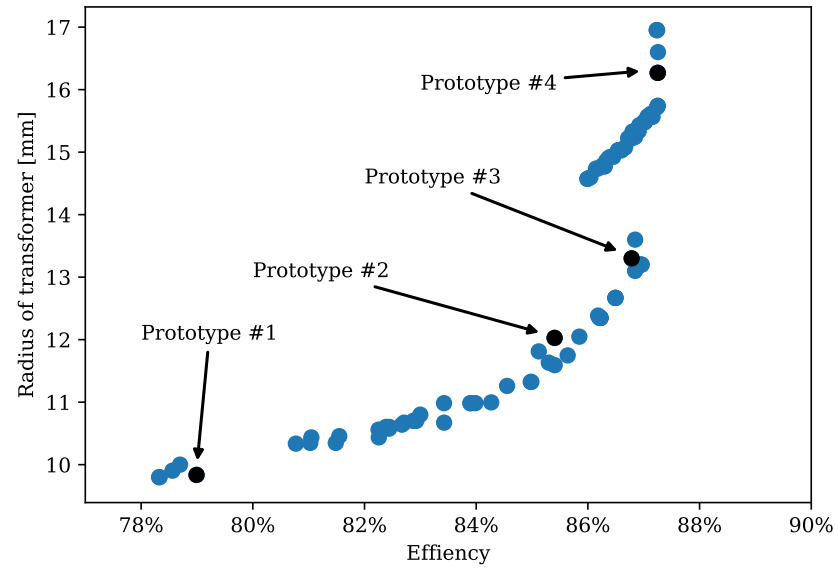

(b)

Fig. 9. Developed and identified Pareto front using the genetic algorithm. a) Development over several generations. The later generations move down right and form a Pareto front. b) The last generation showing the Pareto front and the realised prototypes.

TABLE V

AVerage Values of Key Parameters of the Pareto Front that is Plotted in Fig. 9B.

\begin{tabular}{cccccccccc}
$N_{\text {prim }}: N_{\text {sec }}$ & $\begin{array}{c}w_{0} \\
w_{\text {sec }}\end{array}$ & $\begin{array}{c}L_{s} \\
\mathrm{MHz}\end{array}$ & $\begin{array}{c}L_{\text {prim }} \\
\mathrm{MHz}\end{array}$ & $\begin{array}{c}L_{\text {sec }} \\
\mathrm{nH}\end{array}$ & $\begin{array}{c}C_{p} \\
\mathrm{nH}\end{array}$ & $\begin{array}{c}C_{s} \\
\mathrm{nH}\end{array}$ & $\begin{array}{c}C_{\text {rect }} \\
\mathrm{pF}\end{array}$ & $\begin{array}{c}\text { coupling } \\
\mathrm{pF}\end{array}$ & \\
\hline $4: 4$ & 7.28 & 10.64 & 460 & 419 & 431 & 1049 & 1310 & 540 & 0.62
\end{tabular}

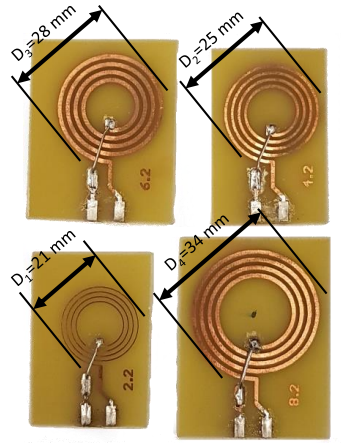

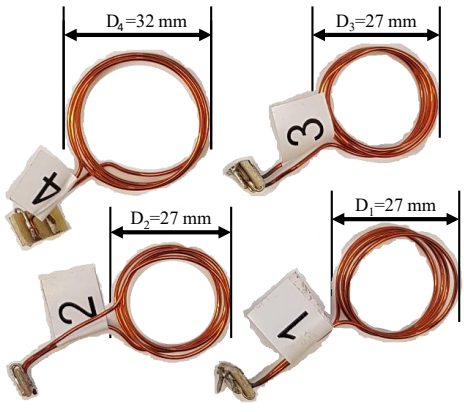

(a)

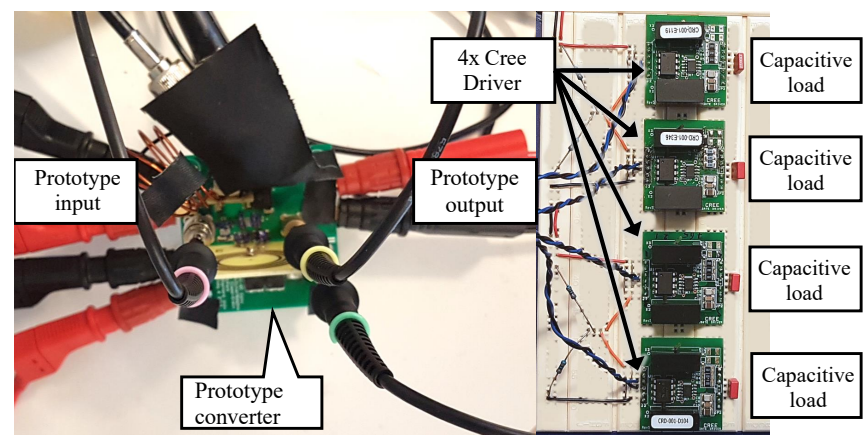

(b)

Fig. 10. a) Transformers and series inductors for the four prototypes used in the experimental validation. b) Photo of prototype 4 and the load on the output consisting of four Cree SiC MOSFET drivers (CRD-001).

\section{EXPERIMENTAL VALIDATION}

From the final generation, several individuals are chosen to verify the simulation results. A multitude of prototypes serves to show that the simulation model is in fact a good representation for the actual circuit behaviour. Four individuals are chosen based on their total distance from all other individuals, excluding the outliers on each side. Similarly to the initial prototype, the air transformers are made on PCBs which are seen, together with the series inductors, $L_{s}$, in Fig. 10a. Each series inductor was manually tuned to obtain the targeted inductance value. This method is time consuming, however, a more industrial product could use $\mathrm{PCB}$ printed or 3D printed toroids [46], [47]. An added benefit of the toroid structure will be the containment of the field inside the structure. The measured component values and the error compared to the simulated values are shown for all four prototypes in Table VI. The inductance values are within $6 \%$ deviation from the simulated value, while the coupling coefficient is within $\pm 1 \%$. The capacitance values of the prototypes have little deviation compared to simulation as the capacitors are selected based on their measured capacitance value using the impedance analyser.

The efficiency and output voltage of the four prototypes are shown in Fig. 11. Figure 11a shows the change in output voltage as a function of the output power and the characteristic nose curve is observed. The minimum output power or current is limited thermally during testing as the inverter case temperature comes close to its maximum allowable value when operating without a heatsink. Figure $11 \mathrm{~b}$ shows the efficiency as a function of output power. However, this way 


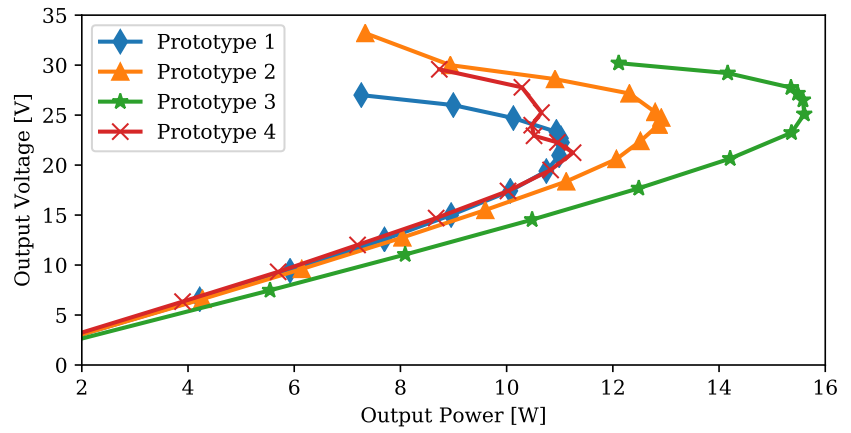

(a)

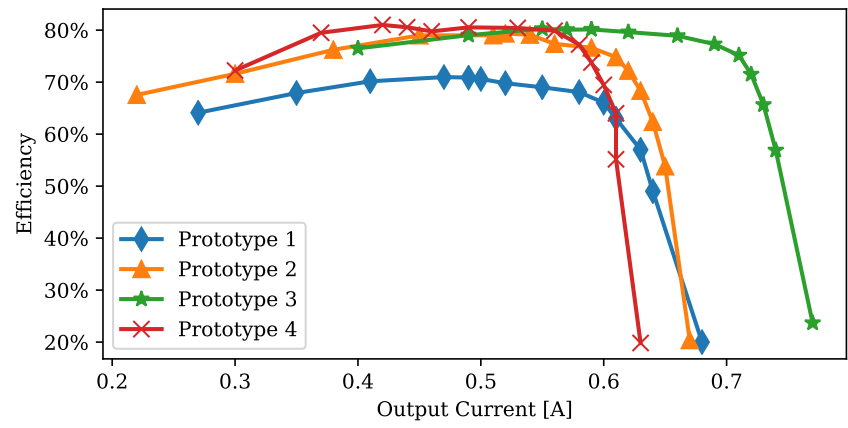

(c)

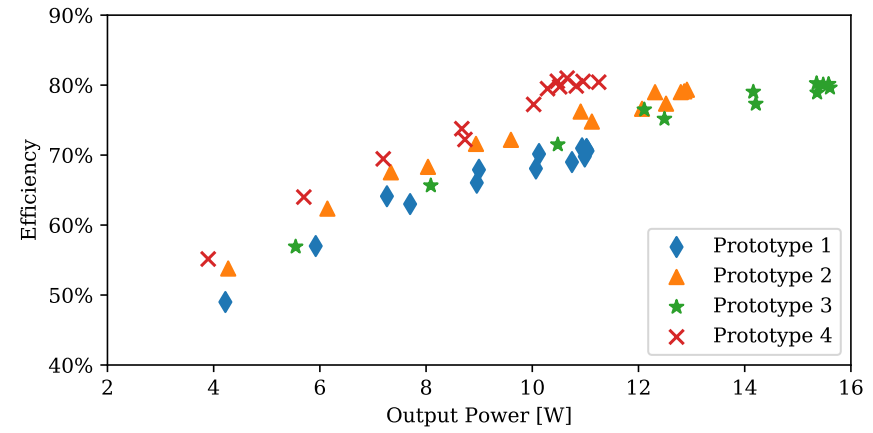

(b)

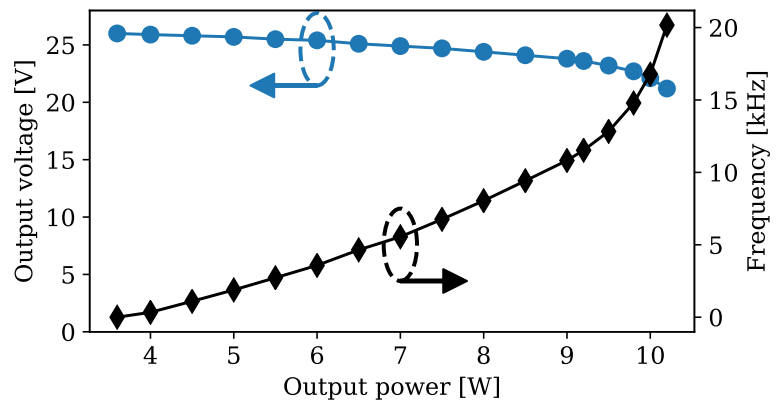

(d)

Fig. 11. Experimental results from the four prototypes. a) Efficiency as a function of output power for varying load resistance. b) Output voltage variation as a function of output. c) Efficiency as a function of output current. d) The load line of prototype \#4 while powering four Cree SiC MOSFET drivers with capacitive loads, where the output power varies with the driver switching frequency.

TABLE VI

COMPARISON OF COMPONENT VALUES BETWEEN SIMULATION AND THE EXPERIMENTAL SETUP.

\begin{tabular}{|c|c|c|c|c|c|c|c|c|}
\hline \multirow{3}{*}{$\frac{\text { Prototype }}{L_{s}[\mathrm{nH}]}$} & \multicolumn{7}{|c|}{ Experimental value (error \%) } & \\
\hline & \multicolumn{2}{|l|}{$\# 1$} & \multicolumn{2}{|c|}{$\# 2$} & \multicolumn{2}{|l|}{ \#3 } & \multicolumn{2}{|l|}{$\# 4$} \\
\hline & 468 & $(1 \%)$ & 501 & $(1 \%)$ & 502 & $(2 \%)$ & 469 & $(2 \%)$ \\
\hline$L_{\text {prim }}[\mathrm{nH}]$ & 395 & $(3 \%)$ & 392 & $(5 \%)$ & 388 & $(6 \%)$ & 561 & $(4 \%)$ \\
\hline$L_{\text {sec }}[\mathrm{nH}]$ & 395 & $(4 \%)$ & 380 & $(6 \%)$ & 377 & $(6 \%)$ & 573 & $(5 \%)$ \\
\hline coupling & 0.57 & $(1 \%)$ & 0.64 & $(-1 \%)$ & 0.68 & $(<1 \%)$ & 0.71 & $(-1 \%)$ \\
\hline$C_{p}[\mathrm{pF}]$ & 724 & $(<1 \%)$ & 1036 & $(<1 \%)$ & 1075 & $(4 \%)$ & 1170 & $(3 \%)$ \\
\hline$C_{s}[\mathrm{pF}]$ & 1347 & $(<1 \%)$ & 1379 & $(-1 \%)$ & 1376 & $(-1 \%)$ & 1199 & $(-1 \%)$ \\
\hline$C_{\text {rect }}[\mathrm{pF}]$ & 859 & $(2 \%)$ & 808 & $(<1 \%)$ & 813 & $(1 \%)$ & 232 & $(-1 \%)$ \\
\hline$C_{\text {coupling }}(\operatorname{sim} / \exp )[\mathrm{pF}]$ & $4.5 / 7$. & & $8.1 / 11$ & & $10.7 / 1$ & & $13.8 / 18$ & \\
\hline
\end{tabular}

of presenting the efficiency leads to double points per output power. To present the data without double points, the efficiency is plotted as a function of output current in Fig. 11c, where zero current equals an open circuit condition and the maximum value equals a short circuit condition.

The maximum efficiency is measured to be $71.0 \%, 79.3 \%$, $80.3 \%, 81.0 \%$, ordered according to the prototype number and, hence, with increasing transformer size. All prototypes are able to supply over $10 \mathrm{~W}$, with prototype \#3 being able to supply more than $15 \mathrm{~W}$. This fits well with the previously discussed application of the auxiliary power supply. Compared to [15], [16], the proposed designs have the same or better efficiency while increasing both the output power and the air gap height.
The latter results in an increased insulating voltage of the isolated auxiliary power supply. The measured efficiency of the prototypes are lower than the values found in simulation $(79.0 \%, 85.4 \%, 86.8 \%, 87.3 \%)$. The difference in efficiency between simulation and measured for prototypes \#2-4 is approximately $6-7 \%$. As shown with the initial prototype, increased losses should be expected for the experimental setup due to the modelling of the inverter. As discussed in Section III, the simulation model consistently underestimated the losses in the inverter due to the phenomena of dynamic on-resistance of GaN HEMTs. The additional inverter losses measured for all prototypes accounts for the missing percentage points in the measured efficiency compared to the 
simulated efficiency. The larger efficiency gap of prototype \#1 is likely related to the thin track width of prototype \#1. The track width of this individual is only $0.2 \mathrm{~mm}$, making the performance sensitive to small variations in track width due to the manufacturing resolution of the in-house etching technique. This is verified as the measured resistance values of the PCB transformer are found to be $15 \%$ higher than simulated. The converters appear to loose their soft switching operation when the load goes towards an open circuit. Other practical converter constraints like nose curve shape, inverter temperature and soft switching operation should be included in future optimisations either as separate simulations or by simplified analytic expressions.

The main difference between prototypes \#1-3 is the track width, which only slightly decreases the transformer inductance and winding resistance while the coupling slightly increases. Prototype \#1 differs from the other prototypes by having a resonance of the primary side higher than the operating frequency $(8.65 \mathrm{MHz})$ whereas prototypes \#2-4 are close to the operating frequency $(6.99,6.85$, and $6.79 \mathrm{MHz}$, respectively). For the secondary side, prototypes \#1-3 are similar $(8.18,8.57,8.58 \mathrm{MHz}$, respectively) whereas prototype \#4 has a larger resonance frequency of $11.54 \mathrm{MHz}$. The maximum output power and variations in the output voltage with load resistance are observed to change for different design configurations. Desired converter characteristics could be added to the optimisation algorithm to further develop the design space in the desired direction. It is hypothesised that the shapes of the nose curves of prototypes \#1-3 are similar due to similar impedance values on the rectifier side. Their differences in resistance and coupling gives the premise for the maximum power output and efficiency.

The coupling capacitance of the transformer is indirectly included in the optimisation algorithm since the transformer area is minimised. The coupling capacitance was modelled using FEMM and measured experimentally for the four prototypes. Simulated and measured values are given in Table VII. From measurements, it is found that the coupling capacitances are in the range of 8 to $14 \mathrm{pF}$, which is higher than compared to the literature [15], [16]. Part of this capacitance is related to the higher value of $\epsilon_{r}$ of FR4 that is used as the dielectric material in this work. All measured values are 3 to $5 \mathrm{pF}$ higher than the simulated values due to the simplified geometry of the simulation model and possibly the exact value of $\epsilon_{r}$ for the prototypes. In view of these aspects, the measured values correspond well to the simulation. The coupling capacitance appears to be proportional to the area of the transformer windings, as can be expected. The optimisation algorithm could be modified by adding a simulation of the coupling capacitance to calculation this value directly. Although, minimisation of the transformer size, in particular the surface area, also minimises the coupling capacitance. Similarly, increasing the distance between the primary and secondary side also decreases the coupling capacitance.

Prototype 4 is tested in a setup that is similar to the target application. The setup is shown in Fig. 10b, where the prototype power supply provides power to four Cree $\mathrm{SiC}$ MOSFET drivers (CRD-001). Each driver is loaded with a
$220 \mathrm{nF}$ capacitor, which is equivalent to the gate capacitance of modern $3.3 \mathrm{kV} \mathrm{SiC} \mathrm{MOSFET} \mathrm{high} \mathrm{power} \mathrm{module.} \mathrm{The}$ switching frequency of the driver load is varied from 1 to $20 \mathrm{kHz}$ to vary the loading of the prototype converter. The resulting load line of the prototype converter is shown in Fig. 11d. A drop in the output voltage from 26 to $21.2 \mathrm{~V}$ is observed over the whole power range. However, the output voltage at $9 \mathrm{~W}$ is only $23.8 \mathrm{~V}$, a voltage drop of less than $10 \%$.

Based on the transformer geometry of the experimental prototypes, a comparison is made with similar works regarding the insulation voltage. The comparison is summarised in Table VII. For this work, the breakdown voltage of the insulation barrier is estimated using published experimental data for different materials and material thicknesses combined with the gap distances between the primary and secondary side [16]. For the prototypes, the insulating material is FR4, which results in a breakdown voltage of $32 \mathrm{kV}_{\text {peak }}$. If polyesterimide or Teflon material of $1.5 \mathrm{~mm}$ thickness is used instead, breakdown voltages of approximately 48 and $80 \mathrm{kV}$ are obtained. Furthermore, the dielectric constant of these materials, 3.5 [48] and 2.0 [49] respectively, would result in a decreased coupling capacitance down to a half with respect to the case of FR4. This results in coupling capacitances in the range of $4-9 \mathrm{pF}$ for the prototypes in this work.

\section{CONCLUSION}

This paper presents the design and optimisation of a suitable topology for an isolated DC-DC auxiliary power supply with high isolation voltage and low coupling capacitance. The converter consists of a GaN HEMT inverter operating at $6.78 \mathrm{MHz}$, an LCC resonance tank, a coreless transformer and a class $\mathrm{E}$ low $\mathrm{dv} / \mathrm{dt}$ rectifier. In regard to the application, the converter is suitable for supplying gate drivers in medium voltage converters. A design methodology is developed, however, SPICE investigations show that optimal designs might lie outside the validity of the design equations. In particular, the rectifier shows preferential characteristics when designed with a resonance frequency higher than the operating frequency $\left(\omega_{\text {res }} \geq 1.6\right)$; the output voltage regulation improves and the inductor size is reduced. A virtual prototyping tool is developed based on a genetic algorithm that is used in an holistic optimisation of the converter. The optimisation algorithm maximises the converter efficiency while minimising the coupling capacitance. Using the developed Pareto front, the best performing prototype exhibits a peak efficiency of $81 \%$ when supplying $15 \mathrm{~W}$ of power. The prototypes all have smaller transformer sizes, and hence, lower coupling capacitance compared to the existing design method in literature [27]. Moreover, the coupling capacitances of the prototypes are found through measurement in the range of 7 to $18 \mathrm{pF}$. However, if a more suitable dielectric material like Teflon is used, the coupling capacitance is halved and coupling capacitances below $10 \mathrm{pF}$ can be obtained. At the same time, using Teflon as insulation material could increase the isolation voltage up to $80 \mathrm{kV}$. 
TABLE VII

EVALUATION OF THE ISOLATION VOLTAGE AND TRANSFORMER GEOMETRY COMPARED TO OTHER PUBLISHED WORK.

\begin{tabular}{crrrrrr} 
Publication & $\begin{array}{c}\text { Gap } \\
\text { distance } \\
{[\mathrm{mm}]}\end{array}$ & $\begin{array}{r}\text { Radius - } \\
\text { ferrite or winding } \\
{[\mathrm{mm}]}\end{array}$ & $\begin{array}{r}\text { Radius - } \\
\text { dielectric } \\
{[\mathrm{mm}]}\end{array}$ & $\begin{array}{r}\text { Peak } \\
\text { efficiency }\end{array}$ & $\begin{array}{r}\text { Isolation } \\
\text { voltage } \\
{\left[\mathrm{kV}_{\text {peak }}\right]}\end{array}$ & $\begin{array}{r}\text { Insulation } \\
\text { material }\end{array}$ \\
\hline$[16]$ & 1.0 & 7 & 50 & $70 \%$ & 40 & Teflon \\
\hline$[10]$ & 13 & 40 & 86 & $90 \%$ & 95 & Polyurethane \\
\hline This work & 1.5 & $10-17$ & - & $71-81 \%$ & $* 48$ & Polyesterimide \\
& & & & $* 80$ & Teflon \\
\hline
\end{tabular}

* Peak insulation voltage is estimated using the experimental data on breakdown voltages for different materials and material thicknesses found in [16].

\section{APPENDIX}

This section shows a numerical example using the analytical design method given in Section II. For the example, the input voltage is set to $48 \mathrm{~V}$ while the desired output voltage is chosen to $20 \mathrm{~V}$ for the maximum load requirement of $10 \mathrm{~W}$. Firstly, the rectifier passive components are found according to (1), (2) and (3).

$$
\begin{gathered}
R_{\text {load,min }}=\frac{(20 \mathrm{~V})^{2}}{10}=40 \Omega \\
L_{\text {sec }}=\frac{40 \Omega}{\omega_{0} \cdot 0.3884}=2.418 \mu \mathrm{H} \\
C_{\text {rect }}=\frac{1}{\omega_{0}^{2} L_{\text {sec }}}=0.2279 \mathrm{nF}
\end{gathered}
$$

where $\omega_{0}$ equals $2 \pi \cdot 6.78 \mathrm{MHz}$ and the values of $Q_{r}$ and $M_{V}$ are given as 0.3884 and 0.3684 in [27] for the maximum output-power capability of the rectifier. Then, the input voltage is found using (4):

$$
V_{m}=\frac{20 \mathrm{~V}}{0.3684}=54.29 \mathrm{~V}
$$

In the following step, $C_{p}$ is found. However, this requires that the coupling factor of the transformer is known. For the example, it is assumed that the factor is 0.6. Furthermore, $L_{\text {prim }}$ is assumed equal to $L_{\text {sec }}$. From (13), $C_{p}$ becomes:

$$
C_{p}=\frac{\pi \cdot 54.29 \mathrm{~V}}{\sqrt{2} \cdot \omega_{0}^{2} \cdot 0.6 \cdot 2.418 \mu \mathrm{H} \cdot 48 \mathrm{~V}}=0.9545 \mathrm{nF}
$$

The corresponding $I_{\text {prim }}$ is found to be 0.88 A. Using (11), $L_{s}$ evaluates to:

$$
L_{s}=\frac{1}{\omega_{0}^{2} \cdot 0.9545 \mathrm{nF}}=0.577 \mu \mathrm{H}
$$

The values of $L_{i}$ is set as $0.814 \cdot L$ [27]. With a target switching current of $-1.25 \mathrm{~A}$, the series capacitor is calculated according to (17):

$$
\begin{aligned}
C_{s} & =\frac{0.9545 \mathrm{nF}}{\frac{\pi \cdot 24.59 \Omega \cdot(-1.25 \mathrm{~A})}{\sqrt{2} \cdot 48 \mathrm{~V}}+\frac{2.418 \mu \mathrm{H}}{0.577 \mu \mathrm{H}}+\frac{-46.27 \Omega}{24.59 \Omega}-\frac{\pi^{2}}{8}} \\
& =0.471 \mathrm{nF}
\end{aligned}
$$

The component values are evaluated using SPICE simulations for modelling the reference circuit [27]. Moreover:

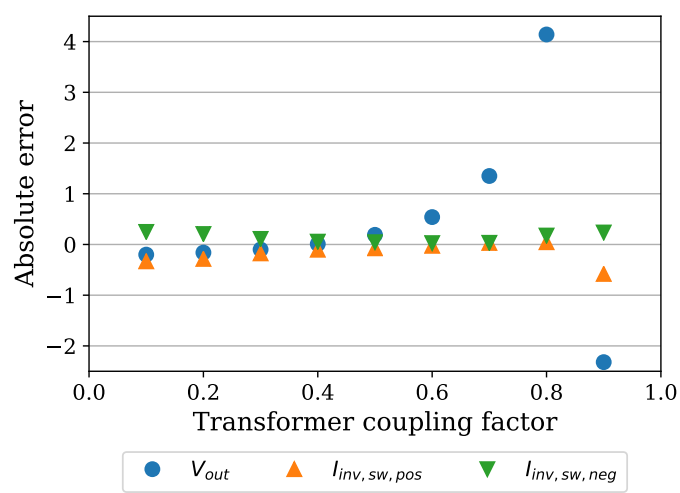

Fig. 12. Absolute error between the proposed topology and the reference circuit for the output voltage and the inverter current at the switching instant, $I_{i n v, s w}$, for either falling ( $\left.p o s\right)$ or rising (neg) edge of the inverter output voltage.

- The input source, $V_{i n}$, is a trapezoidal voltage that changes between 0 and $48 \mathrm{~V}$ with rise and fall times of 10 ns.

- The diode has no junction capacitance, and is modelled with $R_{o n}$ and $R_{o f f}$ of $50 \mathrm{~m} \Omega$ and $10 \mathrm{M} \Omega$, respectively.

- All passive components have zero resistance.

For the design case, the output voltage of the full circuit evaluates to $21.11 \mathrm{~V}$ compared to $20.57 \mathrm{~V}$ for the reference circuit, an error of $2.6 \%$. The error for changing transformer coupling is shown in Fig. 12. The error in output voltage is small for a coupling factors up to approximately 0.7. Above this value, the second harmonic generated by the rectifier influences the converter behaviour and the calculations become less accurate. Nevertheless, the coupling factor of coreless transformers are usually lower than 0.7. Additionally, it is seen that the switching current also has a slight offset meaning that tweaking of the $C_{s}$ value is likely needed for the final converter design. Moreover, the error between the positive and negative current is asymmetric due to the second harmonic content in the inverter current.

Figure 13 shows the behaviour of the rectifier output with changing load condition. The increasing voltage and power with increasing load resistance are characteristics of a current source. In many cases, a voltage source behaviour is preferred over the current source behaviour. 


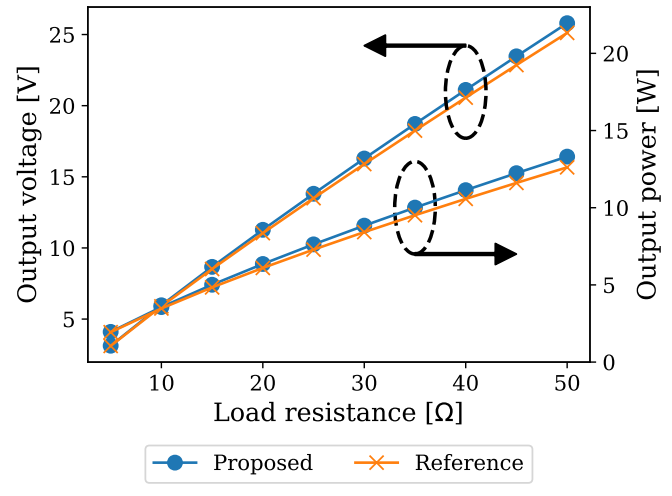

Fig. 13. The inverter output voltage and power as a function of load resistance.

\section{ACKNOWLEDGMENTS}

This work is the result of research stays at both G2Elab, Grenoble, France and Stanford University, Stanford, USA, for which both institutions are thanked.

\section{REFERENCES}

[1] H. Abu-Rub, J. Holtz, J. Rodriguez, and G. Baoming, "Medium-voltage multilevel converters - state of the art, challenges, and requirements in industrial applications," IEEE Trans. Ind. Electron., vol. 57, no. 8, pp. 2581-2596, Aug. 2010.

[2] J. Rodriguez, S. Bernet, B. Wu, J. O. Pontt, and S. Kouro, "Multilevel voltage-source-converter topologies for industrial medium-voltage drives," IEEE Trans. Ind. Electron., vol. 54, no. 6, pp. 2930-2945, Dec. 2007.

[3] H. Polinder, J. A. Ferreira, B. B. Jensen, A. B. Abrahamsen, K. Atallah, and R. A. McMahon, "Trends in wind turbine generator systems," IEEE J. Emerg. Sel. Top. Power Electron., vol. 1, no. 3, pp. 174-185, Sep. 2013.

[4] M. E. Baran and N. R. Mahajan, "DC distribution for industrial systems: Opportunities and challenges," IEEE Trans. Ind. Appl., vol. 39, no. 6, pp. 1596-1601, Nov. 2003.

[5] G. F. Reed, B. M. Grainger, A. R. Sparacino, and Z. Mao, "Ship to grid: Medium-voltage dc concepts in theory and practice," IEEE Power Energy Mag., vol. 10, no. 6, pp. 70-79, Nov. 2012.

[6] F. Mura and R. W. D. Doncker, "Design aspects of a medium-voltage direct current (MVDC) grid for a university campus," in Proc. 8th Int. Conf. on Power Electron. (ECCE Asia '11), May 2011, pp. 2359-2366.

[7] A. Giannakis and D. Peftitsis, "MVDC distribution grids and potential applications: Future trends and protection challenges," in Proc. 20th European Conf. Power Electron. and Appl. (EPE'18 ECCE Europe), Sep. 2018, pp. P.1-P.9.

[8] K. Sharifabadi, L. Harnefors, H.-P. Nee, S. Norrga, and R. Teodorescu, Design, Control, and Application of Modular Multilevel Converters for HVDC Transmission Systems. John Wiley \& Sons, 2016.

[9] T. Modeer, S. Norrga, and H. Nee, "High-Voltage Tapped-Inductor Buck Converter Utilizing an Autonomous High-Side Switch," IEEE Transactions on Industrial Electronics, vol. 62, no. 5, pp. 2868-2878, May 2015.

[10] B. Wunsch, D. Zhelev, and B. Oedegard, "Externally-fed auxiliary power supply of MMC converter cells," in Proc. 18th European Conf. Power Electron. and Appl. (EPE'16 ECCE Europe), Sep. 2016, pp. 1-10.

[11] V. Nguyen, P. Lefranc, and J. Crebier, "Gate driver supply architectures for common mode conducted EMI reduction in series connection of multiple power devices," IEEE Trans. Power Electron., vol. 33, no. 12, pp. 10265-10276, Dec. 2018.

[12] D. Peftitsis, M. Antivachis, and J. Biela, "Auxiliary power supply for medium-voltage modular multilevel converters," in Proc. 17th European Conf. Power Electron. and Appl. (EPE'15 ECCE Europe), Sep. 2015, pp. 1-11.

[13] J. Gottschlich, M. Schäfer, M. Neubert, and R. W. D. Doncker, "A galvanically isolated gate driver with low coupling capacitance for medium voltage SiC MOSFETs," in Proc. 18th European Conf. Power Electron. and Appl. (EPE'16 ECCE Europe), Sep. 2016, pp. 1-8.
[14] J. Hu, J. Wang, R. Burgos, and D. Boroyevich, "Current-Transformer Based Gate-Drive Power Supply with Reinforced Isolation," in 2018 IEEE Energy Conversion Congress and Exposition (ECCE), Sep. 2018, pp. 6328-6335.

[15] S. Am, P. Lefranc, D. Frey, and R. Hanna, "Design methodology for very high insulation voltage capabilities power transmission function for IGBT gate drivers based on a virtual prototyping tool," IET Power Electron., vol. 10, no. 5, pp. 545-554, 2017.

[16] B. Sarrazin, R. Hanna, P. Lefranc, S. Am, F. Dumas, and J. P. Lavieville, "Insulated power supply for gate drivers up to $40 \mathrm{kV}$ for medium-voltage direct current applications," IET Power Electron., vol. 10, no. 15, pp. 2143-2148, 2017.

[17] K. Mainali, S. Madhusoodhanan, A. Tripathi, K. Vechalapu, A. De, and S. Bhattacharya, "Design and evaluation of isolated gate driver power supply for medium voltage converter applications," in Proc. IEEE Applied Power Electron. Conf. and Expo. (APEC'16), Mar. 2016, pp. 1632-1639.

[18] T. Batra, G. Gohil, A. K. Sesham, N. Rodriguez, and S. Bhattacharya, "Isolation design considerations for power supply of medium voltage silicon carbide gate drivers," in Proc. IEEE Energy Conversion Congr. and Expo. (ECCE'17), Oct. 2017, pp. 2552-2559.

[19] H. Fujita, "A Resonant Gate-Drive Circuit With Optically Isolated Control Signal and Power Supply for Fast-Switching and High-Voltage Power Semiconductor Devices," IEEE Trans. Power Electron., vol. 28, pp. 5423-5430, 2013.

[20] X. Zhang, H. Li, J. A. Brothers, L. Fu, M. Perales, J. Wu, and J. Wang, "A Gate Drive With Power Over Fiber-Based Isolated Power Supply and Comprehensive Protection Functions for 15-kV SiC MOSFET," IEEE Journal of Emerging and Selected Topics in Power Electronics, vol. 4, no. 3, pp. 946-955, Sep. 2016.

[21] N. Rouger, D. Colin, L. T. Le, and J. C. Crébier, "CMOS gate drivers with integrated optical interfaces for extremely fast power transistors," in 2016 International Conference on Electrical Systems for Aircraft, Railway, Ship Propulsion and Road Vehicles International Transportation Electrification Conference (ESARS-ITEC), Nov. 2016, pp. 1-6.

[22] K. Kusaka, K. Orikawa, J. i Itoh, K. Morita, and K. Hirao, "Isolation system with wireless power transfer for multiple gate driver supplies of a medium voltage inverter," in Proc. Int. Power Electronics Conf. (IPEC-Hiroshima 2014 - ECCE Asia), May 2014, pp. 191-198.

[23] M. Takasaki, Y. Miura, and T. Ise, "Wireless power transfer system for gate power supplies of modular multilevel converters," in Proc. 8th IEEE Int. Power Electronics and Motion Control Conf. (IPEMC'16 ECCE Asia), May 2016, pp. 3183-3190.

[24] C.-J. Lim and S. Park, "Isolated power supply for multiple gate drivers using wireless power transfer system with single-antenna receiver," $J$. Power Electron., vol. 17, no. 5, pp. 1382-1390, 2017.

[25] B. Wunsch, J. Bradshaw, I. Stevanović, F. Canales, W. Van-der-Merwe, and D. Cottet, "Inductive power transfer for auxiliary power of medium voltage converters," in Proc. IEEE Applied Power Electron. Conf. and Expo. (APEC'15), Mar. 2015, pp. 2551-2556.

[26] C.-S. Wang, G. A. Covic, and O. H. Stielau, "Power transfer capability and bifurcation phenomena of loosely coupled inductive power transfer systems," IEEE Trans. Ind. Electron., vol. 51, no. 1, pp. 148-157, Feb. 2004.

[27] M. K. Kazimierczuk and D. Czarkowski, Resonant Power Converters. John Wiley \& Sons, Inc, 1995.

[28] A. Ivascu, M. K. Kazimierczuk, and S. Birca-Galateanu, "Class E resonant low dv/dt rectifier," IEEE Trans. Circuits Syst. Fundam. Theory Appl., vol. 39, no. 8, pp. 604-613, Aug. 1992.

[29] G. Kkelis, D. C. Yates, and P. D. Mitcheson, "Class-E half-wave zero $\mathrm{dv} / \mathrm{dt}$ rectifiers for inductive power transfer," IEEE Trans. Power Electron., vol. 32, no. 11, pp. 8322-8337, Nov. 2017.

[30] M. Liu, M. Fu, and C. Ma, "A compact class E rectifier for megahertz wireless power transfer," in Proc. IEEE PELS Workshop on Emerging Technologies: Wireless Power (WoW'15), Jun. 2015, pp. 1-5.

[31] M. Liu, Y. Qiao, S. Liu, and C. Ma, "Analysis and design of a robust class $\mathrm{E}^{2} \mathrm{dc}-\mathrm{dc}$ converter for megahertz wireless power transfer," IEEE Trans. Power Electron., vol. 32, no. 4, pp. 2835-2845, Apr. 2017.

[32] T. Nagashima, X. Wei, E. Bou, E. Alarcón, M. K. Kazimierczuk, and H. Sekiya, "Analysis and design of loosely inductive coupled wireless power transfer system based on class- $\mathrm{E}^{2} \mathrm{dc}-\mathrm{dc}$ converter for efficiency enhancement," IEEE Trans. Circuits Syst. Regul. Pap., vol. 62, no. 11, pp. 2781-2791, Nov. 2015

[33] J. M. Rivas, O. Leitermann, Y. Han, and D. J. Perreault, "A very high frequency dc-dc converter based on a class $\boldsymbol{\Phi}_{2}$ resonant inverter," IEEE Trans. Power Electron., vol. 26, no. 10, pp. 2980-2992, Oct. 2011. 
[34] Z. Pantic, S. Bai, and S. M. Lukic, "ZCS $L C C$-compensated resonant inverter for inductive-power-transfer application," IEEE Trans. Ind. Electron., vol. 58, no. 8, pp. 3500-3510, Aug. 2011.

[35] LMG5200 80V, 10A GaN Half-Bridge Power Stage, Texas Instruments, Mar. 2017, rev. D.

[36] K. Li, P. Evans, and M. Johnson, "SiC/GaN power semiconductor devices: A theoretical comparison and experimental evaluation under different switching conditions," IET Electr. Syst. Transp., vol. 8, no. 1, pp. 3-11, 2018.

[37] O. C. Spro, D. Peftitsis, O. M. Midtgard, and T. Undeland, "Modelling and quantification of power losses due to dynamic on-state resistance of GaN E-mode HEMT," in Proc. IEEE 18th Workshop Control and Modeling for Power Electron. (COMPEL'17), Jul. 2017, pp. 1-6.

[38] Y. Cai, A. J. Forsyth, and R. Todd, "Impact of GaN HEMT dynamic onstate resistance on converter performance," in Proc. IEEE Applied Power Electron. Conf. and Expo. (APEC'17), Mar. 2017, pp. 1689-1694.

[39] G. Zulauf, S. Park, W. Liang, K. Surakitbovorn, and J. M. R. Davila, " $\mathrm{C}_{\text {oss }}$ losses in $600 \mathrm{~V} \mathrm{GaN}$ power semiconductors in soft-switched, high- and very-high-frequency power converters," IEEE Trans. Power Electron., vol. 33, no. 12, pp. 10748 - 10763, 2018.

[40] B. H. Waters, B. J. Mahoney, G. Lee, and J. R. Smith, "Optimal coil size ratios for wireless power transfer applications," in 2014 IEEE International Symposium on Circuits and Systems (ISCAS), Jun. 2014 pp. 2045-2048.

[41] D. Meeker, Finite Element Magnetic Modelling (FEMM), Available: http://www.femm.info.

[42] Jia Zhuang, Grayson Zulauf, and Juan Rivas-Davila, "Substrate bias effect on E-mode GaN-on-Si HEMT Coss losses," in Proc. 6th IEEE Workshop Wide Bandgap Power Devices and Appl. (WiPDA'18), Atlanta GA, USA, Sep. 2018, pp. 130-133.

[43] R. Li, X. Wu, S. Yang, and K. Sheng, "Dynamic on-state resistance test and evaluation of GaN power devices under hard- and soft-switching conditions by double and multiple pulses," IEEE Trans. Power Electron., vol. 34, no. 2, pp. 1044-1053, Feb. 2019.

[44] B. J. Galapon, A. J. Hanson, and D. J. Perreault, "Measuring dynamic on resistance in GaN transistors at $\mathrm{MHz}$ frequencies," in Proc. IEEE 19th Workshop Control and Modeling for Power Electron. (COMPEL'18), Jun. 2018, pp. 1-8.

[45] K. Deb, A. Pratap, S. Agarwal, and T. Meyarivan, "A fast and elitist multiobjective genetic algorithm: NSGA-II," IEEE Trans. Evol. Comput., vol. 6, no. 2, pp. 182-197, Apr. 2002.

[46] C. R. Sullivan, W. Li, S. Prabhakaran, and S. Lu, "Design and fabrication of low-loss toroidal air-core inductors," in Proc. IEEE Power Electron. Specialists Conf. (PESC'07), Jun. 2007, pp. 1754-1759.

[47] W. Liang, L. Raymond, and J. Rivas, "3D printed air core inductors for high frequency power converters," in Proc. IEEE Energy Conversion Congr. and Expo. (ECCE'14), Sep. 2014, pp. 971-979.

[48] L. Fetouhi, J. Martinez-Vega, and B. Petitgas, "Electric conductivity, aging and chemical degradation of polyesterimide resins used in the impregnation of rotating machines," IEEE Trans. Dielectr. Electr. Insul., vol. 25, no. 1, pp. 294-305, Feb. 2018.

[49] P. Ehrlich, "Dielectric properties of teflon from room temperature to $314^{\circ} \mathrm{C}$ and from frequencies of $10^{2}$ to $10^{5} \mathrm{c} / \mathrm{s}, " J$. Res. Natl. Bur. Stand. vol. 51, no. 4, p. 185 , Oct. 1953.

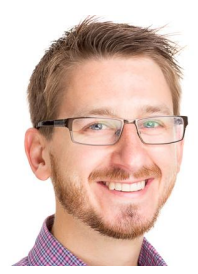

Ole Christian Spro (S'17) was born in Høvik, Norway in 1986. He received the M.Sc. degree in electrical power engineering from the Norwegian University of Science and Technology, Trondheim, Norway, in 2013. He is currently pursuing his $\mathrm{PhD}$ at the same university on the design of power converters using GaN HEMTs in high frequency applications.

From 2013 til 2016 he was with SINTEF Energy Research, Trondheim, where he was mainly working ity of power electronic components.

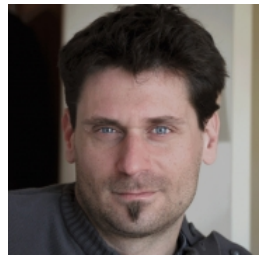

Pierre Lefranc received the M.S. degree from Supélec, Paris, France, and PARIS XI, Orsay, France, in 2002. He received the Ph.D. degree in electrical engineering from the Institut National de Sciences Appliquées de Lyon, Villeurbanne, France, in 2005. He was an Assistant Professor in the Energy Department of the Supélec E3S engineering school (France) from 2006 to 2012 . He is currently with the ENSE3 Engineering School as Associate Professor at INP Grenoble in the G2ELAB laboratory, Grenoble, France. His research interests include modeling, optimization, design of power electronic applications and gate driver for semiconductor devices.

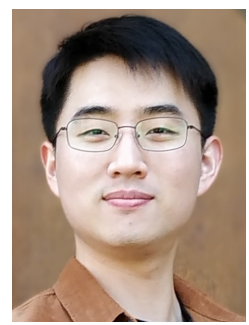

Sanghyeon Park (S'16) was born in Seoul, Korea, in 1991. He received the B.S. degree in electrical engineering from Seoul National University, Seoul, Korea, in 2015, and the M.S. degree in electrical engineering from Stanford University, Stanford, CA, USA, in 2019, where he is currently working toward the Ph.D. degree in electrical engineering. His research interest lies on high-frequency high-voltage power converters.

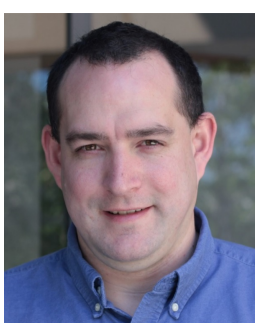

Juan Rivas-Davila (S'01-M'06-SM'17) was born in Mexico City, Mexico. He received the B.A.Sc. degree from the Monterrey Institute of Technology, Monterrey, Mexico, in 1998, and the S.M. and Sc.D. degrees from the Laboratory of Electromagnetic and Electronic Systems, Massachusetts Institute of Technology, Cambridge, MA, USA, in 2003 and 2006, respectively. From 2007 to 2011, he was a Power Electronics Engineer with the HighFrequency Power Electronics Group at the General Electric Global Research Center, Niskayuna, NY, USA. From 2011 to 2013, he was an Assistant Professor at the University of Michigan, Ann Arbor, MI, USA. In 2014, he joined Stanford University, Stanford, CA, USA, as an Assistant Professor in the Electrical Engineering Department. His research interests include power electronics, RF power amplifiers, resonant converters, soft-switching topologies, and the design of air-core passive components for VHF power conversion. 


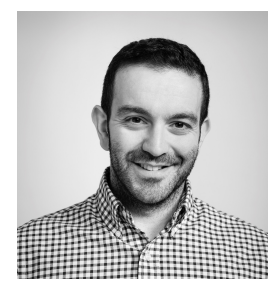

Dimosthenis Peftitsis (S'03-M'13-SM'15) was born in Kavala, Greece, in 1985. He received the Diploma degree (Hons.) in electrical and computer engineering from the Democritus University of Thrace, Xanthi, Greece, in 2008 and the Ph.D. degree from the KTH Royal Institute of Technology, Stockholm, Sweden, in 2013. In 2008, he was with the ABB Corporate Research, Västerås, Sweden, for six months, where he was involved in the diploma thesis. From 2013 to 2014, he was a Postdoctoral Researcher involved in the research on $\mathrm{SiC}$ converters at the Department of Electrical Energy Conversion, KTH Royal Institute of Technology. From 2014-2016, he was working as a Postdoctoral Fellow at the Lab for High Power Electronics Systems, ETH Zurich, where he is involved in dc-breakers for multiterminal HVDC systems. In May 2016, he joined the Norwegian University of Science and Technology in Trondheim, Norway, as an Associate Professor of power electronics at the Department of Electrical Power Engineering. His current research interests are in the area of power converters design using WBG devices (e.g. $\mathrm{SiC}, \mathrm{GaN}$ ) including gate and base drive circuits and dc-breaker design for MV and HVDC systems. In addition to these, Prof. Peftitsis' research also focuses on reliability assessment and lifetime modelling of high-power semiconductor devices, including reliability of $\mathrm{SiC}$ power switches.

Prof. Peftitsis is currently a member of the EPE International Scientific Committee and also serving as the chairman of the Norway IEEE joint PELS/IAS/IES chapter.

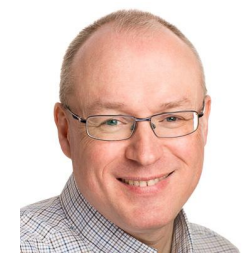

Ole-Morten Midtgård (M'03) was born in 1967. He received the M.Sc. and Ph.D. degrees in Electric Power Engineering from the Norwegian University of Science and Technology (NTNU) in 1992 and 1997, respectively. He is currently Head of Department at NTNU's Department of Electric Power Engineering, where he has been a Professor since 2012. $\mathrm{He}$ has broad research interests, which in addition to Power Electronics include integration of PV in Smart Grids, and Electromagnetic Computations.

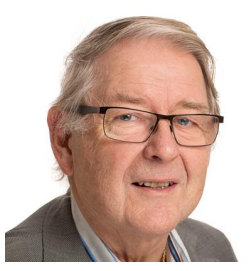

Tore Undeland (M'86-SM'92-F'00) was born in Bergen, Norway, in 1945. He received the M.Sc. and Ph.D. degrees from the Norwegian University of Science and Technology (NTNU), Trondheim, Norway, in 1970 and 1977, respectively. Since 1970, he has been with NTNU, where he held the position of Full Professor since 1984. Since 2013, he holds the position as Professor Emeritus. He has also served as an Adjunct Professor with Chalmers University of Technology, Gothenburg, Sweden, since 2000. He is a coauthor of the well-known textbook Power Electronics: Converters, Applications, and Design (Wiley, 1989, 1995, and 2003). His research interests include power converters, snubbers, and control in power electronics and has authored and coauthored more than 200 papers.

Prof. Undeland was the Chairman of the EPE'97 Conference in Trondheim, has served as the President of the European Power Electronics and Drives Association (EPE) and is a member of the Norwegian Academy of Technological Sciences. He was active in the IEEE Power Electronics Society, where he also has been a Distinguished Lecturer. Prof. Undeland has been a IEEE Fellow since 2001. 